\title{
Plateaus in the Hall Resistance Curve at Filling Factors $2<v<3$
}

\author{
Shosuke Sasaki \\ KYOKUGEN (Center for Quantum Science and Technology under Extreme Conditions), Osaka University, \\ 1-3 Machikaneyama, Toyonaka, Osaka 560-8531, Japan \\ Correspondence should be addressed to Shosuke Sasaki; sasaki@ns.sist.ac.jp
}

Received 15 October 2013; Accepted 11 November 2013; Published 19 February 2014

Academic Editors: S. Bud'ko, H. Eisaki, and V. Gasparian

Copyright (C) 2014 Shosuke Sasaki. This is an open access article distributed under the Creative Commons Attribution License, which permits unrestricted use, distribution, and reproduction in any medium, provided the original work is properly cited.

The fractional quantum Hall (FQH) states with higher Landau levels have new characters different from those with $0<v<2$. The $\mathrm{FQH}$ states at $2<v<3$ are examined by developing the Tao-Thouless theory. We can find a unique configuration of electrons with the minimum Coulomb energy in the Landau orbitals. Therein the electron (or hole) pairs placed in the first and second nearest Landau orbitals can transfer to all the empty (or filled) orbitals at $v_{0}=8 / 3,14 / 5,7 / 3,11 / 5$, and 5/2 via the Coulomb interaction. More distant electron (or hole) pairs with the same centre position have the same total momentum. Therefore, these pairs can also transfer to all the empty (or filled) orbitals. The sum of the pair energies from these quantum transitions yields a minimum at $\nu=v_{0}$. The spectrum of the pair energy takes the lowest value at $v_{0}$ and a higher value with a gap in the neighbourhood of $v_{0}$ because many transitions are forbidden at a deviated filling factor from $\nu_{0}$. From the theoretical result, the FQH states with $v=v_{0}$ are stable and the plateaus appear at the specific filling factors $v_{0}$.

\section{Introduction}

The plateau at the filling factor $v=5 / 2$ attracts a great deal of attention because of a new fractional quantum Hall (FQH) character. The plateau in the filling factor $v>2$ has characters different from that at $0<v<2$. For example, Pan et al. [1] have found a deep minimum of the diagonal resistance, $R_{X X}$ and $R_{Y Y}$, at $\nu=5 / 2$ and $7 / 2$. At $v=9 / 2$ and $11 / 2$ the diagonal resistance exhibits a strongly anisotropic behaviour, where $R_{X X}$ has a sharp peak while $R_{Y Y}$ has a minimum at $v=9 / 2,11 / 2[1-3]$. (The definition of the coordinate axes, $x, y$, and $z$, will be shown in Figure 5 of the next section.) Eisenstein et al. [4] have obtained the plateaus of Hall resistance $R_{X Y}$ at $v=5 / 2$ and $7 / 2$ with even denominator. Furthermore, the other plateaus have been discovered at $v=$ $7 / 3,8 / 3,11 / 5,14 / 5,16 / 5$, and $19 / 5$ with odd denominator as seen in Figure 1.

The plateaus have the precise Hall resistance value. For example, the plateau at $\nu=7 / 2$ has the Hall resistance value $2 h /\left(7 e^{2}\right)$ within $0.015 \%$ as measured in [4]. This accuracy of the Hall resistance indicates that the $v=(7 / 2)$ state has a lower energy than the one at $v=(7 / 2)(1 \pm 0.00015)$.
Further experimental data are shown in Figure 2 which have been observed by Dean et al. [5] and Xia et al. [6] The Hall resistance-curve in the left panel of Figure 2 [5] is different from that in the right panel [6]. This difference means that the shape of the Hall resistance versus magnetic field curve depends on the samples and the experimental conditions (magnetic field strength, etc.). In particular, the difference is large at $\nu=16 / 7,11 / 5$.

When the magnetic field is tilted from the direction perpendicular to the quasi-2D electron system, the Hall resistance plateau at $v=5 / 2$ disappears as seen in Figure 3 which has been found by Csáthy et al. [7]. On the other hand, the $v=7 / 3,8 / 3$ plateaus persist with the tilt as in Figure 3 .

The temperature dependence of $R_{X X}$ has been measured by many researchers. For example, the temperature dependence of the diagonal resistance curves has been measured by Pan et al. [8]. The diagonal resistance curve at $36 \mathrm{mK}$ is different from that at $6 \mathrm{mK}$. Furthermore, some local minima in the diagonal resistance curve disappear at $36 \mathrm{mK}$. Using the temperature dependence of $R_{X X}$, the Arrhenius plots are drawn to give an energy gap. The energy gap is shown in 

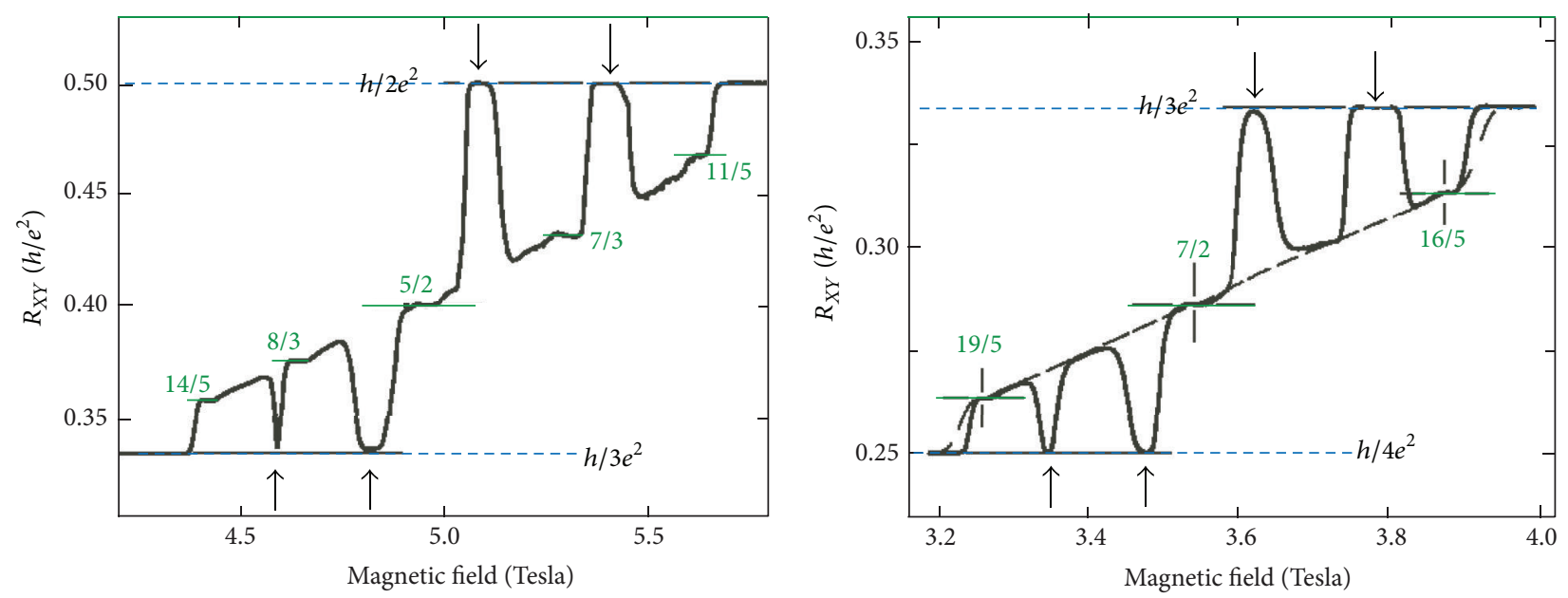

FIGURE 1: Behavior of Hall resistance in the region of $2<v<4$ quoted from [4].

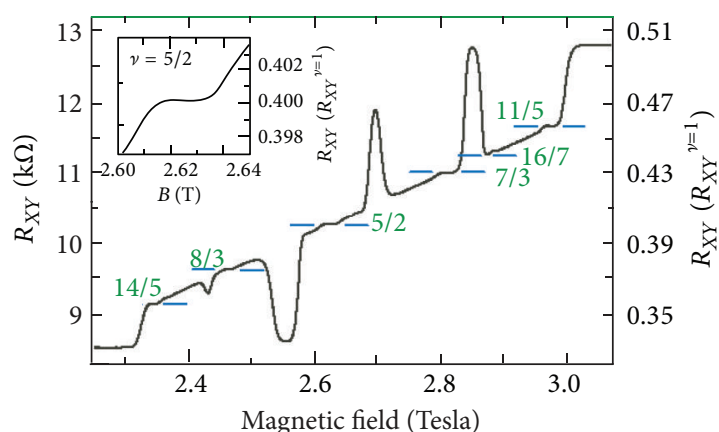

(a)

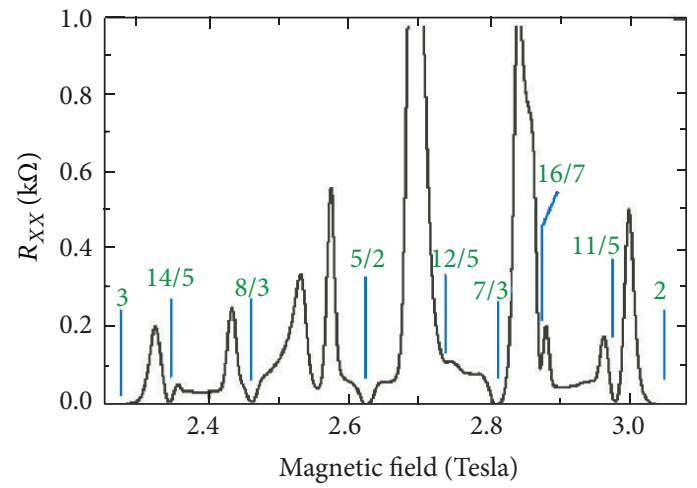

(b)

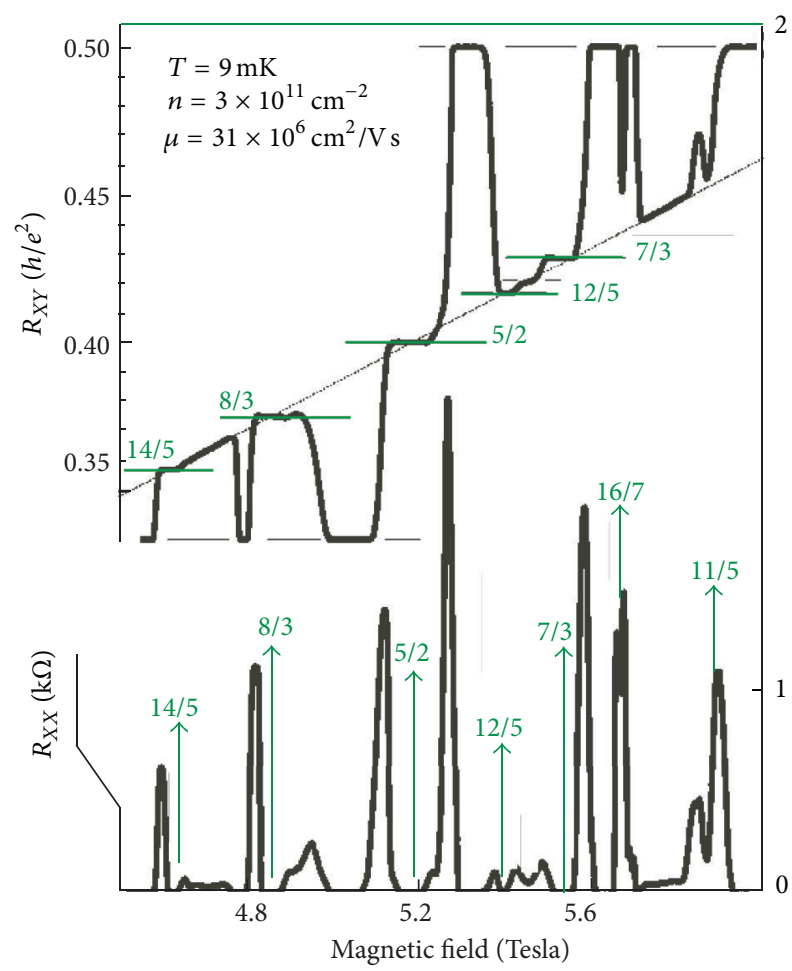

(c)

FIGURE 2: Hall resistance curve in the region $2<v<3$. The left panel is quoted from [5] and the right panel from [6].

Figure 4 which is obtained by Choi et al. [9] and also in the paper [5].

The data obtained in the high mobility sample [9] give the energy gap for the filling factors of $14 / 5,19 / 7,8 / 3,5 / 2,7 / 3$, $16 / 7$, and $11 / 5$ as in Figure 4.

These experimental findings at $v>2$ have stimulated theoretical studies. Several theories have been proposed to explain the plateaus of the Hall resistance at $v>2$, especially at $v=5 / 2$. Some of them are briefly reviewed below.
Koulakov et al. have studied the ground state of a partially filled upper Landau level in a weak magnetic field. They have used the effective interaction [10] which was derived by Aleiner and Glazman in the 2D-electron system with high Landau levels, taking into account the screening effect by the lower fully occupied levels. Then, they have found that the ground state is a charge-density wave (CDW) state with a large period [11]. Moessner and Chalker studied a $2 \mathrm{D}$-electron system with a fermion hardcore interaction 


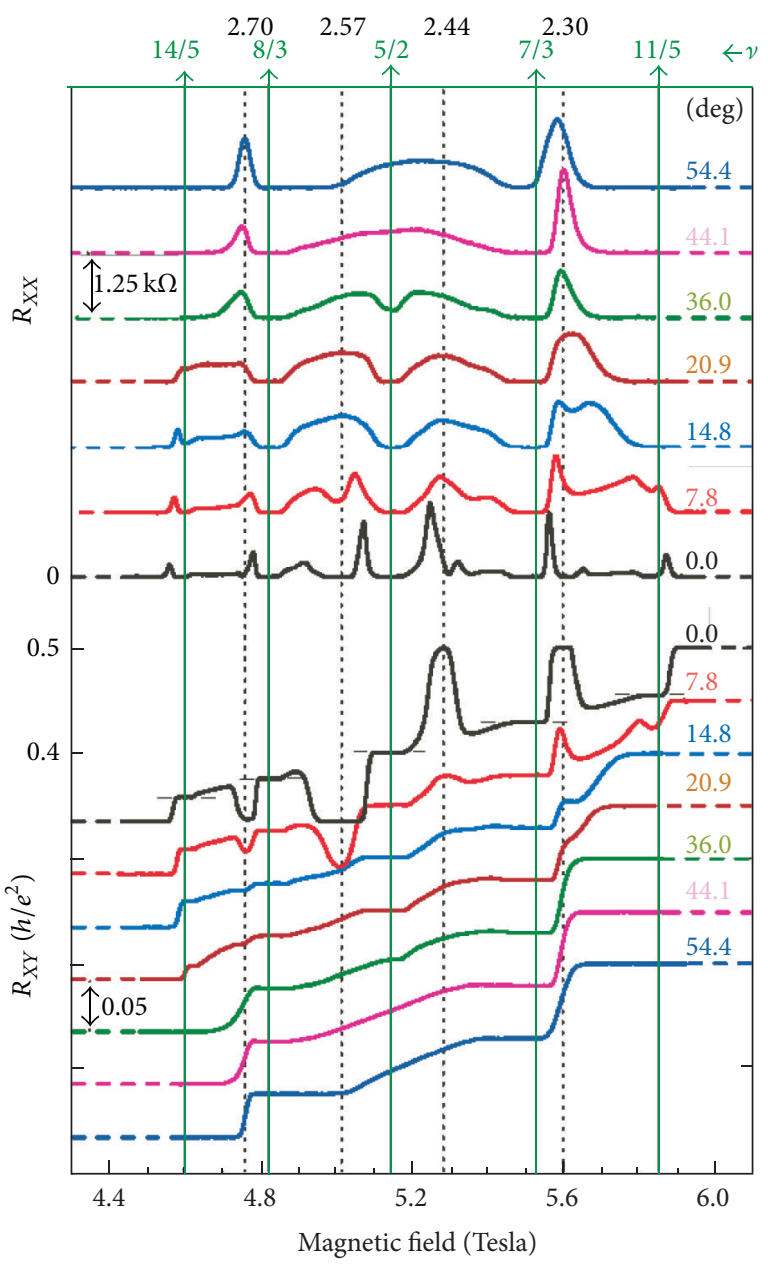

FIgURE 3: Tilt dependence of the Hall resistance and diagonal resistance [7].

and without disorder. They found a transition to both unidirectional and triangular charge-density wave states at finite temperatures [12]. Rezayi et al. numerically studied a 2D-electron system in magnetic field with a high Landau level half filled by electrons. In finite size systems with up to 12 electrons and torus geometry, they found a chargedensity wave ordering in the ground state. Their results show that the highest weight single Slater determinant has the occupation pattern 11111000001111100000, where 1 and 0 stand, respectively, for an occupied orbital and an empty orbital [13].

Haldane and Rezayi investigated the pair state with spinsinglet [14]. They used a hollow core Hamiltonian. In the Landau level number $L=1$, the hollow core Hamiltonian has the first pseudopotential $V_{1}>0$ although the zeroth Haldane pseudopotential $V_{0}$ is zero. They found a ground state called HR state. Moore and Read were inspired by the structure of the HR state, and constructed the pair state a $p$-wave $\left(p_{x}-i p_{y}\right)$ polarized state. They have described the FQH state in terms of conformal-field-theory [15]. The state is called the MooreRead state (MR state). In [16], Read wrote "the wavefunction $\psi_{\mathrm{MR}}$ represents BCS $[17,18]$ pairing of composite fermions.

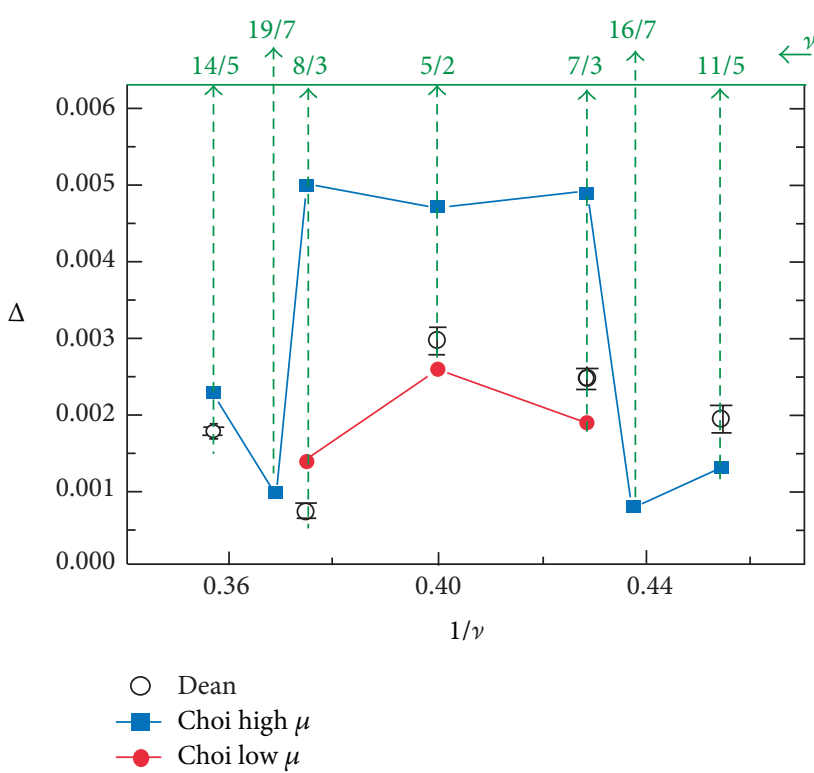

FIgURE 4: Energy gaps for the FQH states. Open circles are quoted from [5]. Solid circles and squares are quoted from [9].

One type are the charged vortices discussed above, with charge $1 /(2 q)$ which according to MR are supposed to obey nonabelian statistics." Greiter et al. investigated the MR state from the viewpoint of the composite fermion pair $[19,20]$. The statistics are an ordinary abelian fractional statistics.

Morf argued the quantum Hall states at $v=5 / 2$ by a numerical diagonalization [21]. He studied spin-polarized and -unpolarized states with $N \leq 18$ electrons. His result indicates that the $5 / 2$ state is expected to be the spinpolarized MR state. Rezayi and Haldane [22] confirmed Morf's results. Their results are based on numerical studies for up to 16 electrons in two geometries: sphere and torus. They found a first order phase transition from a striped state to a strongly paired state. They examined 12 electrons in a rectangular unit cell with the aspect ratio 0.5 . They found the stripe state, the probability weight of which is $58 \%$ for the single Slater determinant state with the occupation pattern 000011110000111100001111 . Also, they found an evidence that the $v=5 / 2$ state is derived from a paired state which is closely related to the MR polarized state or, more precisely, to the state obtained particle-hole $(\mathrm{PH})$ symmetrisation of the MR state [22].

Tao and Thouless $[23,24]$ investigated the FQH states in which the Landau states with the lowest energy are partially filled with electrons. Thus, the theory does not assume any quasiparticle. The present author has developed a theory on the FQH states at $0<v<2[25-32]$ by extending the TaoThouless theory. We will apply the theory to the problem of $5 / 2$ plateau in Section 3. The plateaus at $2.5<v<3$ and at $2<v<2.5$ are discussed in Sections 5 and 6, respectively. Before examining this problem, the fundamental properties will be shortly summarized for the quasi-2D electron system below. 


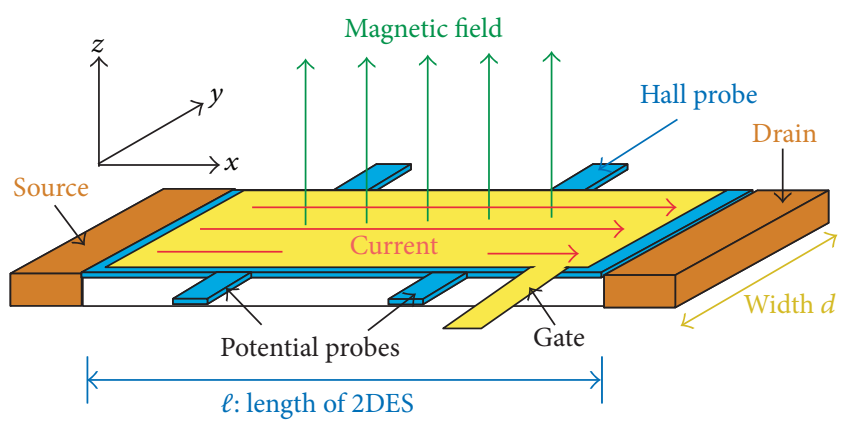

Figure 5: Quantum Hall Device.

\section{The Properties of a Quasi-2D Electron System}

A quantum Hall device is illustrated in Figure 5 where the $x$-axis is the direction of the current and the $y$-axis is the direction of the Hall voltage. Then, the vector potential, A, has the components

$$
\mathbf{A}=(-y B, 0,0),
$$

where $B$ is the strength of the magnetic field. The Hamiltonian, $H_{0}$, of a single electron in the absence of the Coulomb interaction between electrons is given by

$$
H_{0}=\frac{(\mathbf{p}+e \mathbf{A})^{2}}{2 m^{*}}+U(y)+W(z),
$$

where $U(y)$ and $W(z)$ indicate the potentials confining electrons to an ultrathin conducting layer in Figure 5. Therein $m^{*}$ is an effective mass of electron and $\mathbf{p}=\left(p_{x}, p_{y}, p_{z}\right)$ is the electron momentum. The Landau wave function of the single electron is given by

$$
\begin{gathered}
\psi_{L, J}(x, y, z)=\sqrt{\frac{1}{\ell}} \exp \left(\frac{i p x}{\hbar}\right) u_{L} H_{L}\left(\sqrt{\frac{m^{*} \omega}{\hbar}}\left(y-\alpha_{J}\right)\right) \\
\times \exp \left(-\frac{m^{*} \omega}{2 \hbar}\left(y-\alpha_{J}\right)^{2}\right) \phi(z) \\
\omega=\frac{e B}{m^{*}}
\end{gathered}
$$

where $\phi(z)$ is the wave function of the ground state along the $z$-direction, $H_{L}$ is the Hermite polynomial of $L$ th degree, $u_{L}$ is the normalization constant, and $\ell$ is the length of the quasi-2D electron system as in Figure 5 . The integer $L$ is called Landau level number hereafter.

Because of the periodic boundary condition, the momentum $p$ is given by

$$
p=\left[\frac{2 \pi \hbar}{\ell}\right] \times J
$$

The momentum is related to the value $\alpha_{J}$ in the wave function, (3a), as

$$
\alpha_{J}=\frac{p}{(e B)}=\left[\frac{2 \pi \hbar}{(\ell e B)}\right] J .
$$

The eigenenergy is given by

$$
E_{L, J}=\lambda+U\left(\alpha_{J}\right)+\left(\frac{\hbar e B}{m^{*}}\right)\left(L+\frac{1}{2}\right) \quad(L=0,1,2,3, \ldots),
$$

where $L$ is the Landau level number, $\lambda$ is the ground state energy along the $z$-direction, and $U\left(\alpha_{J}\right)$ is the potential energy in the $y$-direction.

When there are many electrons, the total Hamiltonian is given by

$$
\begin{aligned}
H_{T}= & \sum_{i=1}^{N} H_{0, i} \\
& +\sum_{i=1}^{N-1} \sum_{j>i}^{N} \frac{e^{2}}{4 \pi \varepsilon \sqrt{\left(x_{i}-x_{j}\right)^{2}+\left(y_{i}-y_{j}\right)^{2}+\left(z_{i}-z_{j}\right)^{2}}},
\end{aligned}
$$

where $N$ is the total number of electrons, $\varepsilon$ is the permittivity and $H_{0, i}$ is the single particle Hamiltonian of the $i$ th electron without the Coulomb interaction as

$$
H_{0, i}=\frac{\left(\mathbf{p}_{i}+e \mathbf{A}\right)^{2}}{2 m^{*}}+U\left(y_{i}\right)+W\left(z_{i}\right) .
$$

The many-electron state is characterized by a set of Landau level numbers $L_{1}, L_{2}, \ldots, L_{N}$ and a set of momenta $p_{1}, p_{2}, \ldots$, $p_{N}$. The complete set is composed of the Slater determinant as

$$
\begin{aligned}
& \Psi\left(L_{1}, \ldots, L_{N} ; p_{1}, \ldots, p_{N}\right) \\
& =\frac{1}{\sqrt{N !}}\left|\begin{array}{ccc}
\psi_{L_{1}, p_{1}}\left(x_{1}, y_{1}, z_{1}\right) & \cdots & \psi_{L_{1}, p_{1}}\left(x_{N}, y_{N}, z_{N}\right) \\
\vdots & & \vdots \\
\psi_{L_{N}, p_{N}}\left(x_{1}, y_{1}, z_{1}\right) & \cdots & \psi_{L_{N}, p_{N}}\left(x_{N}, y_{N}, z_{N}\right)
\end{array}\right| .
\end{aligned}
$$

This state is the eigenstate of $\sum_{i=1}^{N} H_{0, i}$. The expectation value of the total Hamiltonian is denoted by $W\left(L_{1}, \ldots, L_{N} ; p_{1}, \ldots\right.$, $\left.p_{N}\right)$ which is given by

$$
\begin{aligned}
W & \left(L_{1}, \ldots, L_{N} ; p_{1}, \ldots, p_{N}\right) \\
& =\sum_{i=1}^{N} E_{L_{i}}\left(p_{i}\right)+C\left(L_{1}, \ldots, L_{N} ; p_{1}, \ldots, p_{N}\right),
\end{aligned}
$$

where $C$ is the expectation value of the Coulomb interaction defined by

$$
\begin{aligned}
C & \left(L_{1}, \ldots, L_{N} ; p_{1}, \ldots, p_{N}\right) \\
= & \int \ldots \int \Psi\left(L_{1}, \ldots, L_{N} ; p_{1}, \ldots, p_{N}\right)^{*} \\
& \times \sum_{i=1}^{N-1} \sum_{j>i}^{N} \frac{e^{2}}{4 \pi \varepsilon \sqrt{\left(x_{i}-x_{j}\right)^{2}+\left(y_{i}-y_{j}\right)^{2}+\left(z_{i}-z_{j}\right)^{2}}} \\
& \times \Psi\left(L_{1}, \ldots, L_{N} ; p_{1}, \ldots, p_{N}\right) d x_{1} d y_{1} d z_{1} \ldots d x_{N} d y_{N} d z_{N} .
\end{aligned}
$$


TABLE 1: Energy gap measured for the fractional quantum Hall states in the second Landau level [9].

\begin{tabular}{llllllrr}
\hline$\nu$ & $v=14 / 5$ & $v=19 / 7$ & $v=8 / 3$ & $v=5 / 2$ & $\nu=7 / 3$ & $v=16 / 7$ & $\nu=11 / 5$ \\
\hline Sample A & $252 \mathrm{mK}$ & $108 \mathrm{mK}$ & $562 \mathrm{mK}$ & $544 \mathrm{mK}$ & $584 \mathrm{mK}$ & $94 \mathrm{mK}$ & $160 \mathrm{mK}$ \\
Sample B & $<60 \mathrm{mK}$ & & $150 \mathrm{mK}$ & $272 \mathrm{mK}$ & $206 \mathrm{mK}$ & $<40 \mathrm{mK}$ \\
\hline
\end{tabular}

Hereafter, we call $C\left(L_{1}, \ldots, L_{N} ; p_{1}, \ldots, p_{N}\right)$ "classical Coulomb energy." We divide the total Hamiltonian $H_{T}$ into two parts $H_{D}$ and $H_{I}$ as follows:

$$
\begin{aligned}
H_{D}=\sum_{L_{1}, \ldots, L_{N}} \sum_{p_{1}, \ldots, p_{N}} & \left|\Psi\left(L_{1}, \ldots, L_{N} ; p_{1}, \ldots, p_{N}\right)\right\rangle \\
& \times W\left(L_{1}, \ldots, L_{N} ; p_{1}, \ldots, p_{N}\right) \\
& \times\left\langle\Psi\left(L_{1}, \ldots, L_{N} ; p_{1}, \ldots, p_{N}\right)\right|, \\
H_{I}=H_{T}-H_{D}, &
\end{aligned}
$$

where $H_{I}$ is composed of the off-diagonal elements only. Accordingly, the total Hamiltonian $H_{T}$ of the quasi-2D electron system is a sum of $H_{D}$ and $H_{I}$ as follows:

$$
H_{T}=H_{D}+H_{I}
$$

The Slater determinant composed of the Landau states is the exact eigenstate of $H_{D}$. So we will examine the residual part, namely, quantum transitions via the off-diagonal parts of the Coulomb interaction.

Because the Coulomb interaction depends only upon the relative coordinate of electrons, the total momentum along the $x$-direction conserves in the quasi-2D electron system. That is to say, the sum of the initial momenta $p_{i}$ and $p_{j}$ is equal to that of the final momenta $p_{i}^{\prime}$ and $p_{j}^{\prime}$ :

$$
p_{i}^{\prime}+p_{j}^{\prime}=p_{i}+p_{j}
$$

Next we discuss the configuration of electrons in the Landau orbitals. The previous article [32] has verified that the most uniform configuration of electrons is uniquely determined for any filling factor except at both ends. The effects of the boundaries may be neglected in a macroscopic system. At $v<1$ the Landau states with the Landau level number $L=0$ are partially occupied by electrons and all the states with $L \geq 1$ are empty. For example, the most uniform configuration for $v=2 / 3$ is constructed by repeating of the unit configuration (filled, empty, filled) as shown in Figure 6. This configuration determines the set of the momenta as

$$
\begin{gathered}
p_{2 n-1}=p_{1}+\left(\frac{2 \pi \hbar}{\ell}\right) \times 3(n-1), \quad n=1,2,3, \ldots, \\
p_{2 n}=p_{1}+\left(\frac{4 \pi \hbar}{\ell}\right)+\left(\frac{2 \pi \hbar}{\ell}\right) \times 3(n-1) \\
\text { for the filling factor } \frac{2}{3},
\end{gathered}
$$

where $p_{1}$ is the minimum value of the momentum.

For an arbitrary filling factor, we can also find the most uniform configuration of electrons in the Landau states.

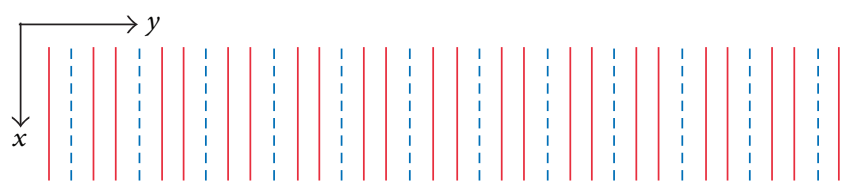

FIgURE 6: Most uniform configuration of electrons at $v=2 / 3$. The current flows along the $x$-axis and the Hall voltage yields along the $y$ axis. Red solid lines indicate the Landau orbitals filled with electron. Blue dashed lines indicate the empty orbitals.

Then, the configuration yields the minimum expectation value of $H_{D}$, namely, the ground state of $H_{D}$.

We next count the number of the Coulomb transitions via $H_{I}$. When the filling factor deviates a little from the specific filling factor, the number of quantum transitions decreases abruptly because of the Fermi-Dirac statistics and the momentum conservation. That is to say, the number of the Coulomb transitions at the specific filling factors takes the largest among those of the neighbouring filling factors. This property produces the minimum energy at the specific filling factors and yields the precise confinement of the Hall resistance. This mechanism can explain the phenomena of the FQHE at $v<2$ without introducing any quasiparticles [2532].

Here, we remark the edge current in the FQH states. Büttiker [33] investigated the current distribution in a 2Delectron system and found the edge current. Both total current and Hall voltage are affected by the edge current in the IQHE but the Hall resistance remains to be the original value. The mechanism has been studied for the FQH states under the existence of the edge current in the article [29]. The precise confinement of the Hall resistance is derived from the momentum conservation along the current direction.

\section{Explanation for the Appearance of 5/2 Plateau}

We first compare the energy gap at $v>2$ with that at $v<$ 2. The energy gap at $v=5 / 2,8 / 3,7 / 3$, and so forth is determined from the experiment [9], the results of which are listed in Table 1 . The energy gap for $v<1$ is shown in Figure 7 which is obtained in [34]. The value of the energy gap changes from sample to sample as in Table 1 . The energy gap at $\nu=2 / 3$ is about $4.3 \mathrm{~K}$ and that of $v=5 / 2$ is about $0.272 \sim 0.544 \mathrm{~K}$ as measured in [9] and [34]. Thus, the energy gap in the region $2<v<3$ is about $1 / 10$ times that in $v<1$. Therefore, we cannot ignore the small terms of various Coulomb transitions in studying the energy spectrum for $v>2$.

In the region of $2<v<3$, all the Landau states with $L=0$ are filled with electrons with up and down spins, 


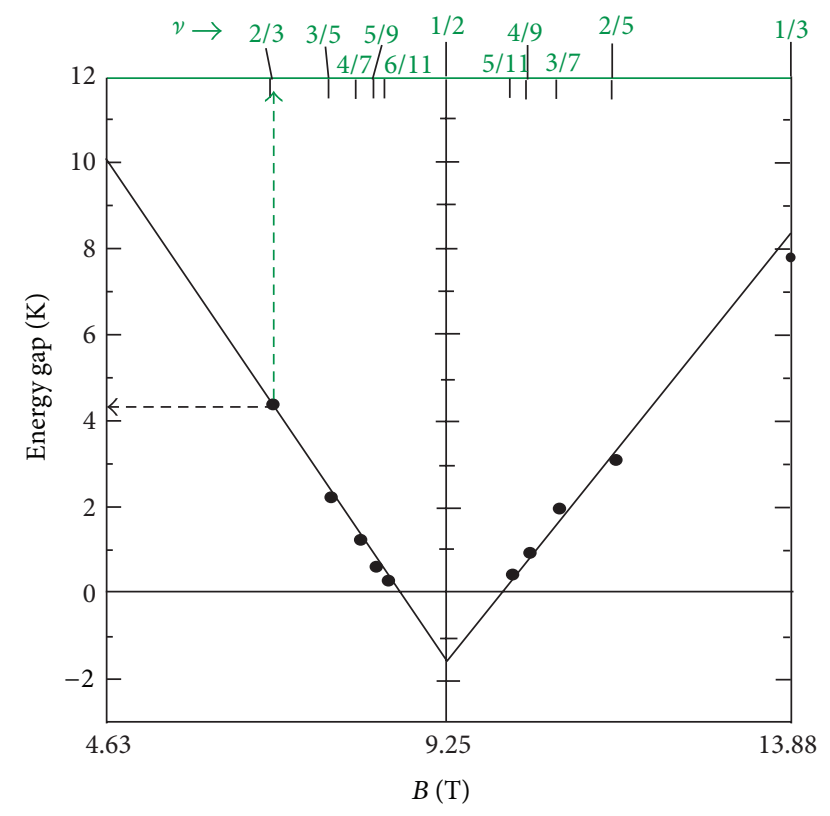

FIGURE 7: Energy gaps at $\nu=2 / 3,3 / 5,4 / 7,5 / 9,6 / 11,5 / 11,4 / 9,3 / 7$, $2 / 5$ in [34].

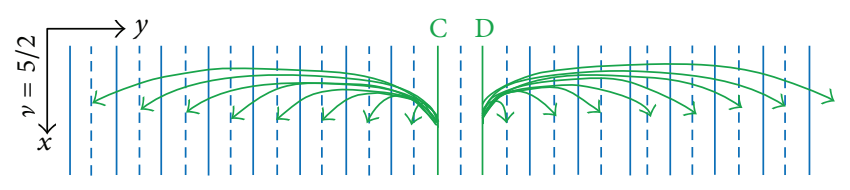

Figure 8: Most uniform configuration at $v=5 / 2$. Dashed lines indicate empty orbitals and solid lines indicate filled orbitals in the second Landau level $L=1$. Allowed transitions are shown by green arrow pairs.

and the Landau states with $L=1$ are partially occupied by electrons. The interactions between electrons depend on the shape of the Landau wave function in the $x$ and $y$ directions, the wave function along the $z$-direction, the screening effect of electrons in the lowest Landau level, and so on. The theories [10-22] reviewed in Section 1 have employed various types of interactions. For example, the first Haldane pseudopotential $V_{1}$ for $L=1$ is positive although $V_{0}$ for the lowest Landau level $(L=0)$ is zero. Thus, the wave function and the interaction in higher Landau levels are different from those in the lowest Landau level.

In the previous articles [25-32], we have ignored the energy from the pairs placed in the second nearest neighboring Landau orbitals, because it is expected to be smaller than that in the first nearest Landau orbitals. For the $v>$ $2 \mathrm{FQH}$ states we have to include the contribution from the electron pairs placed in the second neighboring Landau orbitals because of the small energy-gap as in Table 1.

We first study the $v=5 / 2 \mathrm{FQH}$ state using the method of the previous papers [25-32]. At $\nu=5 / 2=2+1 / 2$, the most uniform configuration of electrons in the $L=1$ level is illustrated in Figure 8 where the fully occupied orbitals with $L=0$ are not drawn for simplicity.
We examine the quantum transitions via the Coulomb interaction $H_{I}$. All the Coulomb transitions satisfy the momentum conservation along the $x$-axis. Figure 8 shows schematically the quantum transitions from the electron pair $\mathrm{CD}$ as an example. The momenta of electrons at $\mathrm{C}$ and $\mathrm{D}$ are described by $p_{\mathrm{C}}$ and $p_{\mathrm{D}}$, respectively. These momenta change to $p_{\mathrm{C}}^{\prime}$ and $p_{\mathrm{D}}^{\prime}$ after the transition. The momentum conservation gives the following relation:

$$
\begin{aligned}
& p_{\mathrm{C}}^{\prime}=p_{\mathrm{C}}-\Delta p, \\
& p_{\mathrm{D}}^{\prime}=p_{\mathrm{D}}+\Delta p,
\end{aligned}
$$

where $\Delta p$ is the momentum transfer. The quantum transition is allowed to empty orbitals only. As seen in Figure 8, the empty orbital exists in the odd numbered orbitals from the left of the orbital C. Therefore, the transfer momentum takes the following value derived from (4) and (5):

$$
\Delta p=\left(\frac{2 \pi \hbar}{\ell}\right) \times(2 n-1) \quad n= \pm 1, \pm 2, \pm 3, \pm 4, \ldots,
$$

where $n=0$ is eliminated because the transition is forbidden by the Pauli exclusion principle. All the allowed transitions are illustrated by the green arrow pairs in Figure 8 . Thus, any electron pair placed in the second neighboring orbitals can also transfer to all the empty orbitals (except $n=0$ ) at $v=5 / 2$.

In order to calculate the pair energies, the following summation $S$ is introduced for the Landau level $L=1$ :

$$
\begin{gathered}
S=-\sum_{\Delta p \neq 0,-4 \pi \hbar / \ell}\left\langle L=1, p_{\mathrm{C}}, p_{\mathrm{D}}\left|H_{I}\right| L=1, p_{\mathrm{C}}^{\prime}, p_{\mathrm{D}}^{\prime}\right\rangle \\
\times \frac{\left\langle L=1, p_{\mathrm{C}}^{\prime}, p_{\mathrm{D}}^{\prime}\left|H_{I}\right| L=1, p_{\mathrm{C}}, p_{\mathrm{D}}\right\rangle}{W_{\mathrm{G}}-W_{\text {excite }}\left(p_{\mathrm{C}} \longrightarrow p_{\mathrm{C}}^{\prime}, p_{\mathrm{D}} \longrightarrow \begin{array}{r}
\left.p_{\mathrm{D}}^{\prime}\right) \\
(18 \mathrm{a})
\end{array}\right.} \\
p_{\mathrm{D}}=p_{\mathrm{C}}+\frac{4 \pi \hbar}{\ell}, \\
p_{\mathrm{C}}^{\prime}=p_{\mathrm{C}}-\Delta p, \quad p_{\mathrm{D}}^{\prime}=p_{\mathrm{D}}+\Delta p .
\end{gathered}
$$

The summation is carried out for all the momentum changes $\Delta p=(2 \pi \hbar / \ell) \times$ integer except $\Delta p=0$ and $-4 \pi \hbar / \ell$. The elimination comes from disappearance of the diagonal matrix element of $H_{I}$. The summation $S$ is positive, because the denominator in (18a) is negative. The perturbation energy $\varsigma_{\mathrm{CD}}$ of the pair CD is expressed by the summation $S$ as follows:

$$
\varsigma_{\mathrm{CD}}=-\left(\frac{1}{2}\right) S
$$

because the function in (18a) is continuous for the argument $\Delta p$ and also the momentum change $2 \pi \hbar / \ell$ is extremely small for a macroscopic size of the device. Therein the factor $1 / 2$ comes from the fact that the number of allowed transitions is equal to the number of the empty orbitals which is half of the total Landau orbitals with $L=1$.

There are many electron pairs like CD. The total number of the pairs like CD is equal to $N_{v=5 / 2}^{L=1}$ which indicates the total number of electrons placed in the Landau orbitals with $L=1$. 
Accordingly, the perturbation energy of all the second nearest electron pairs is given by

$$
E_{\nu=5 / 2}^{\text {pair }}=-\left(\frac{1}{2}\right) S N_{\nu=5 / 2}^{L=1} .
$$

The pair energy per electron is

$$
\frac{E_{\nu=5 / 2}^{\text {pair }}}{N_{\nu=5 / 2}^{L=1}}=-\left(\frac{1}{2}\right) S .
$$

The summation $S$ depends on the thickness, size, and material of the quasi-2D electron system. The reasons are as follows. The wave function along the $z$-axis depends on both thickness and potential shape along the $z$-axis. The wave function length of the $x$-direction depends on the device size. The effective mass of electron and the permittivity depend on the material of the device. Therefore, the classical Coulomb energy $W$ and the transition matrix element vary with changing the quantum Hall device. Accordingly, the value of $S$ varies from sample to sample.

Furthermore, the $L=1$ Landau wave function is zero at its center position because of the Hermite polynomial of $L=$ 1 degree as in (3a). Accordingly, the function form in (18a) is quite different from that for $L=0$. Additionally, we need to consider the screening effect from the $L=0$ electrons. The effect is also unknown. Therefore, we do not go into detail of the summation and treat $S$ as a parameter.

We have ignored the quantum transitions into higher Landau levels with $L \geq 2$. The contribution is extremely small because the excitation energy is very large as follows: the excitation energy from the Landau level with $L$ to that with $L+1$ is given by

$$
E_{L+1}-E_{L}=\frac{\hbar e B}{m^{*}},
$$

which is derived from (6). The effective mass $m^{*}$ differs from material to material and the value in GaAs is about 0.067 times that of free electron. For example, this excitation energy is estimated at the magnetic field strength $4 T$ as

$$
\begin{aligned}
E_{2}-E_{1} & \approx 1.055 \times 10^{-34} \times \frac{1.602 \times 10^{-19} \times 4}{\left(0.067 \times 9.109 \times 10^{-31}\right)} \\
& \approx 1.108 \times 10^{-21}[\mathrm{~J}], \\
\frac{\left(E_{2}-E_{1}\right)}{k_{B}} & \approx 80.3[\mathrm{~K}] \quad \text { for } B=4 T .
\end{aligned}
$$

In the perturbation calculation, the denominator is the energy difference $W_{\mathrm{G}}-W_{\text {excite }}$. When the intermediate state belongs to $L=2$, the main part of $W_{\mathrm{G}}-W_{\text {excite }}$ is $E_{1}-E_{2}$ for $2<v<4$. The value $\left(E_{2}-E_{1}\right) / k_{B}$ is about $80[\mathrm{~K}]$, and so the absolute of the denominator for the intermediate states with $L=2$ is very large compared with that for the intermediate states with $L=1$ in (18a). Therefore, the contribution from the intermediate states with $L \geq 2$ is extremely small, so we ignore them. We examine now the energy gaps in Table 1 which have the magnitude of about $0.1 \mathrm{~K}$. The absolute

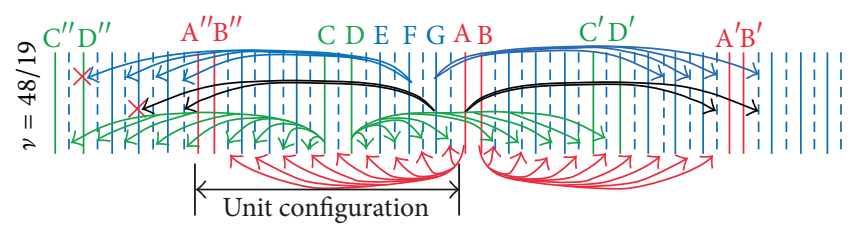

FIGURE 9: Quantum transitions at $\nu=48 / 19$ state.

value of the denominator for the intermediate state with $L=2$ is about 800 times the energy gaps. Accordingly, the intermediate states with $L \geq 2$ may be neglected.

Next, we study the perturbation energy in the neighborhood of $\nu=5 / 2$. As an example, the $\nu=48 / 19=2+(10 / 19)$ state is examined. The most uniform electron configuration is illustrated in Figure 9 where the Landau orbitals with $L=0$ are not shown, for simplicity. The unit configuration is $(1,1,0,1,0,1,0,1,0,1,0,1,0,1,0,1,0,1,0)$, where 1 indicates a filled orbital and 0 an empty orbital.

The electron pair CD can transfer to all the empty orbitals as shown by green arrow pairs. On the other hand the pair GA can transfer to only one site per unit configuration as shown by black arrow pairs in Figure 9 where the red symbol $\times$ indicates the forbidden transition. So the pair energy $\varsigma_{\mathrm{GA}}^{\prime}$ is

$$
\varsigma_{\mathrm{GA}}^{\prime}=-\left(\frac{1}{19}\right) S \quad \text { for } \nu=\frac{48}{19} \text {. }
$$

Therein the coefficient $1 / 19$ indicates that the electron pair GA can transfer to one site per 19 Landau orbitals. The energies of the other pairs are calculated, the results of which are

$$
\begin{gathered}
\varsigma_{\mathrm{FG}}^{\prime}=-\left(\frac{3}{19}\right) S, \quad \varsigma_{\mathrm{EF}}^{\prime}=-\left(\frac{5}{19}\right) S, \\
\varsigma_{\mathrm{DE}}^{\prime}=-\left(\frac{7}{19}\right) S, \quad \varsigma_{\mathrm{CD}}^{\prime}=-\left(\frac{9}{19}\right) S, \quad \text { for } \nu=\frac{48}{19} .
\end{gathered}
$$

We calculate the electron pair $\mathrm{AB}$ which is placed in the nearest neighboring Landau orbitals as in Figure 9. In order to calculate the energy of the nearest pair, we introduce the following summation $T$ :

$$
\begin{aligned}
T=-\sum_{\Delta p \neq 0,-2 \pi \hbar / \ell}\langle L & \left.=1, p_{\mathrm{A}}, p_{\mathrm{B}}\left|H_{I}\right| L=1, p_{\mathrm{A}}^{\prime}, p_{\mathrm{B}}^{\prime}\right\rangle \\
& \times \frac{\left\langle L=1, p_{\mathrm{A}}^{\prime}, p_{\mathrm{B}}^{\prime}\left|H_{I}\right| L=1, p_{\mathrm{A}}, p_{\mathrm{B}}\right\rangle}{W_{\mathrm{G}}-W_{\text {excite }}\left(p_{\mathrm{A}} \longrightarrow p_{\mathrm{A}}^{\prime}, p_{\mathrm{B}} \longrightarrow p_{\mathrm{B}}^{\prime}\right)},
\end{aligned}
$$

where

$$
p_{\mathrm{B}}=p_{\mathrm{A}}+\frac{2 \pi \hbar}{\ell}
$$

which is different from (18b). This summation $T$ is positive because the denominator of (26) is negative. The value $T$ is also treated as a parameter because $T$ varies from sample to sample. The pair AB can transfer to any empty state as shown by the red arrow pairs in Figure 9. The number of the empty 


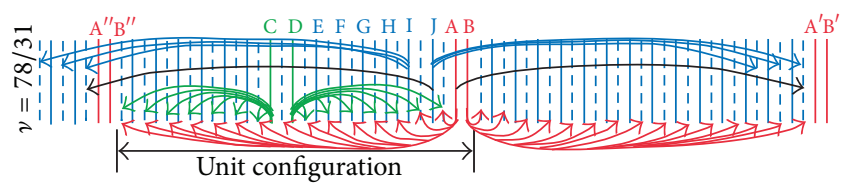

FIGURE 10: Quantum transitions at $v=78 / 31$ state.

states is 9 per unit configuration. Accordingly, the pair energy of $\mathrm{AB}$ is given by

$$
\varsigma_{\mathrm{AB}}^{\prime}=-\left(\frac{9}{19}\right) T, \quad \text { for } \nu=\frac{48}{19} .
$$

The total perturbation energy from all the electron pairs placed in the first and second neighboring Landau orbitals with $L=1$ is

$$
\begin{aligned}
E_{\nu=48 / 19}^{\mathrm{pair}}=\left(\zeta_{\mathrm{AB}}^{\prime}+\zeta_{\mathrm{CD}}^{\prime}+2 \zeta_{\mathrm{DE}}^{\prime}+2 \zeta_{\mathrm{EF}}^{\prime}\right. \\
\left.+2 \zeta_{\mathrm{FG}}^{\prime}+2 \zeta_{\mathrm{GA}}^{\prime}\right)\left(\frac{N_{\nu=48 / 19}^{L=1}}{10}\right) .
\end{aligned}
$$

Substitution of (24), (25), and (28) into (29) yields

$$
E_{\nu=48 / 19}^{\text {pair }}=-\left(\left(\frac{9}{19}\right) T+\left(\frac{41}{19}\right) S\right) \cdot\left(\frac{N_{v=48 / 19}^{L=1}}{10}\right) .
$$

The pair energy per electron for $L=1$ is

$$
\frac{E_{\gamma=48 / 19}^{\text {pair }}}{N_{\gamma=48 / 19}^{L=1}}=-\frac{((9 / 19) T+(41 / 19) S)}{10} .
$$

One more example $v=78 / 31=2+(16 / 31)$ will be examined. The most uniform configuration is shown in Figure 10 where the Landau orbitals with $L=0$ are not shown. The electron pair $\mathrm{CD}$ can transfer to all the empty orbitals as shown by green arrows. On the other hand, the pair JA can transfer to only one site per unit configuration as illustrated by black arrows in Figure 10.

Accordingly, the pair energy $\varsigma_{\mathrm{JA}}^{\prime \prime}$ is

$$
\varsigma_{\mathrm{JA}}^{\prime \prime}=-\left(\frac{1}{31}\right) S \text { for } v=\frac{78}{31} .
$$

The perturbation energies of the other second nearest pairs are given by

$$
\begin{array}{cc}
\varsigma_{\mathrm{IJ}}^{\prime \prime}=-\left(\frac{3}{31}\right) S, & \varsigma_{\mathrm{HI}}^{\prime \prime}=-\left(\frac{5}{31}\right) S, \\
\varsigma_{\mathrm{GH}}^{\prime \prime}=-\left(\frac{7}{31}\right) S, & \varsigma_{\mathrm{FG}}^{\prime \prime}=-\left(\frac{9}{31}\right) S, \\
\varsigma_{\mathrm{EF}}^{\prime \prime}=-\left(\frac{11}{31}\right) S, & \varsigma_{\mathrm{DE}}^{\prime \prime}=-\left(\frac{13}{31}\right) S, \\
\varsigma_{\mathrm{CD}}^{\prime \prime}=-\left(\frac{15}{31}\right) S, & \text { for } \nu=\frac{78}{31} .
\end{array}
$$

The electron pair $\mathrm{AB}$ can transfer to any empty state as shown by the red arrows in Figure 10. The number of the empty states is 15 per unit configuration. Accordingly, the pair energy of $\mathrm{AB}$ is given by

$$
\varsigma_{\mathrm{AB}}^{\prime \prime}=-\left(\frac{15}{31}\right) T \text { for } \nu=\frac{78}{31} .
$$

The total perturbation energy from all the electron pairs placed in the first and second neighboring Landau orbitals with $L=1$ is

$$
\begin{aligned}
E_{\nu=78 / 31}^{\mathrm{pair}}= & \left(\zeta_{\mathrm{AB}}^{\prime \prime}+\zeta_{\mathrm{CD}}^{\prime \prime}+2 \zeta_{\mathrm{DE}}^{\prime \prime}+2 \zeta_{\mathrm{EF}}^{\prime \prime}+2 \zeta_{\mathrm{FG}}^{\prime \prime}\right. \\
& \left.+2 \zeta_{\mathrm{GH}}^{\prime \prime}+2 \zeta_{\mathrm{HI}}^{\prime \prime}+2 \zeta_{\mathrm{IJ}}^{\prime \prime}+2 \zeta_{\mathrm{IA}}^{\prime \prime}\right) \\
& \times\left(\frac{N_{v=78 / 31}^{L=1}}{16}\right) .
\end{aligned}
$$

Substitution of (32), (33), and (34) into (35) yields

$$
E_{v=78 / 31}^{\text {pair }}=-\left(\left(\frac{15}{31}\right) T+\left(\frac{113}{31}\right) S\right) \cdot\left(\frac{N_{v=78 / 31}^{L=1}}{16}\right) .
$$

The pair energy per electron for $L=1$ is

$$
\frac{E_{\nu=78 / 31}^{\text {pair }}}{N_{\nu=78 / 31}^{L=1}}=-\frac{((15 / 31) T+(113 / 31) S)}{16} \text { for } \nu=\frac{78}{31} .
$$

This filling factor $v=78 / 31=2.5161 \cdots$ is close to $v=5 / 2$. The difference between $v=5 / 2$ and $78 / 31$ is about $0.6 \%$. We compare the perturbation energy of the first and second nearest pairs per electron between $v=5 / 2$ and 78/31:

$$
\frac{E_{\nu=5 / 2}^{\text {pair }}}{N_{\nu=5 / 2}^{L=1}}-\frac{E_{\nu=78 / 31}^{\text {pair }}}{N_{\nu=78 / 31}^{L=1}}=(T-9 S)\left(\frac{15}{496}\right) .
$$

From (38) the pair energy at $v=5 / 2$ is lower (or higher) than that at $\nu=78 / 31$ for $T-9 S<0($ or $T-9 S>0)$. We examine the following two Cases 1 and 2.

Case $1(9 S \gg T)$. In this case, the perturbation energy of the first and second pairs per electron is

$$
\frac{E_{\gamma=5 / 2}^{\text {pair }}}{N_{\nu=5 / 2}^{L=1}} \ll \frac{E_{\nu=78 / 31}^{\text {pair }}}{N_{\nu=78 / 31}^{L=1}} .
$$

Accordingly the pair energy at $v=5 / 2$ is sufficiently lower than that in the neighborhood of $v=5 / 2$. So the $v=5 / 2$ state is very stable and the Hall plateau appears at $v=5 / 2$.

Case $2(9 S \ll T)$. In this case, the $v=5 / 2$ Hall plateau does not appear because the pair energy at $\nu=5 / 2$ is higher than that in its neighborhood.

Thus the FQH state is sensitive to the relative value of $S$ and $T$ which is dependent on the materials, thickness of the conducting layer, device structure, and so on. In the next section, we discuss the sample dependent phenomena based on the theory obtained above. 


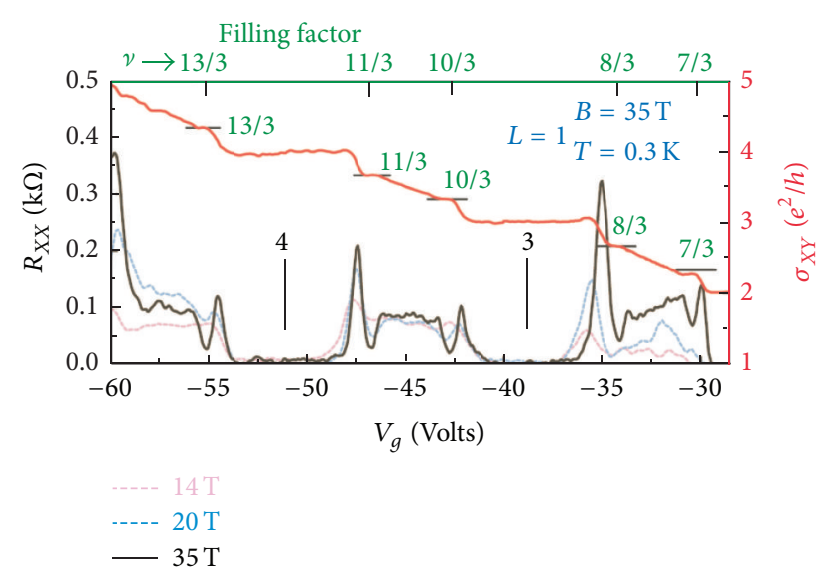

FIGURE 11: Plateaus of Hall conductance and local minima of diagonal resistance in the experimental results of [35].

\section{Sample Dependent Phenomena}

For example, the 5/2 and 7/2 Hall plateaus do not exist on the red curve of Hall conductance obtained by Dean et al. in the article [35] as seen in Figure 11. On the other hand, the experimental results in Figures 1-3 indicate the appearance of the $5 / 2$ and $7 / 2$ Hall plateaus. Thus, the appearance or disappearance of the $v=5 / 2$ and $7 / 2$ plateaus seems to depend upon the samples used in the experiments.

(Note: we point out similar phenomena at $v=1 / 2$. The Hall plateau appears at $v=1 / 2$ in the experimental results [36-40] but disappears in the ordinal experiments for example in the article [34].)

According to our theory examined in Section 3, this property comes from the relative value of $S$ and $T$.

\section{FQH States at Filling Factors $2.5<v<3$}

As will be shown below, in the region of $2.5<v<3$, we find fractional filling factors where both first and second nearest electron pairs can transfer to all the empty Landau orbitals with $L=1$. These filling factors are $v=8 / 3,14 / 5,18 / 7$, and 19/7 for the denominator smaller than nine. The allowed transitions are shown by the arrow pairs in Figures 12, 13, 14, and 15.

Figure 12 shows the most uniform configuration at $v=$ $8 / 3=2+(2 / 3)$ where two types of electron pairs exist. The pair $A B$ represents the first nearest electron pair and the pair $C D$ the second nearest one. Both the pairs $A B$ and $\mathrm{CD}$ can transfer to all the empty orbitals with $L=1$. The allowed transitions are shown by black and green arrow pairs, respectively, in Figure 12.

The number of empty orbitals with $L=1$ is $1 / 3$ of the Landau orbitals with $L=1$. Therefore, the pair energies are given by

$$
\begin{array}{ll}
\varsigma_{\mathrm{AB}}=-\left(\frac{1}{3}\right) T & \text { for } v=\frac{8}{3} \\
\varsigma_{\mathrm{CD}}=-\left(\frac{1}{3}\right) S & \text { for } v=\frac{8}{3}
\end{array}
$$

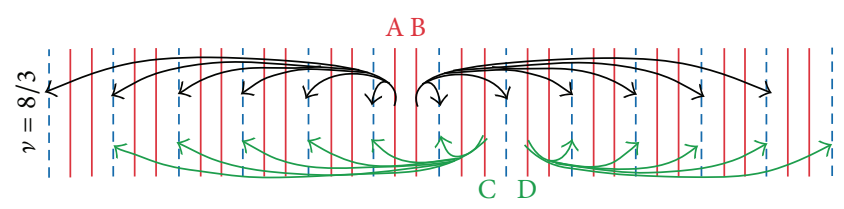

Figure 12: Quantum transitions at $v=8 / 3$ state.

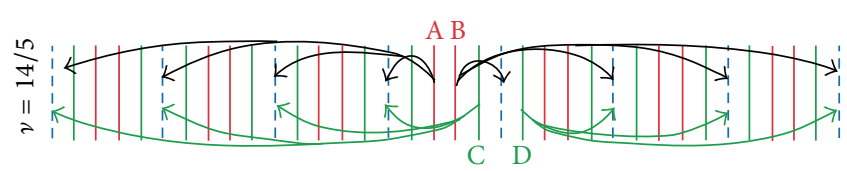

FIGURE 13: Quantum transitions at $\nu=14 / 5$ state.

The total energy of the electron pairs placed in the first and second neighboring Landau orbitals with $L=1$ is

$$
E_{\nu=8 / 3}^{\text {pair }}=\varsigma_{\mathrm{AB}} \times\left(\frac{N_{\nu=8 / 3}^{L=1}}{2}\right)+\varsigma_{\mathrm{CD}} \times\left(\frac{N_{\nu=8 / 3}^{L=1}}{2}\right),
$$

where $N_{v=8 / 3}^{L=1}$ indicates the total number of electrons in the Landau orbitals with $L=1$. Substitution of (40a) and (40b) into (41a) yields

$$
E_{v=8 / 3}^{\text {pair }}=-\left(\frac{T}{6}+\frac{S}{6}\right) N_{\nu=8 / 3}^{L=1}
$$

We examine the pair energy in the limit from the right or left to $v=8 / 3$. Using the same method reported in the previous papers [25-32], we obtain the right and left hand limits as

$$
\begin{gathered}
\lim _{\nu \rightarrow(8 / 3)+\varepsilon} E_{\nu}^{\text {pair }}=-\left(\frac{T}{12}+\frac{S}{12}\right) N_{\nu=8 / 3}^{L=1}, \\
\lim _{v \rightarrow(8 / 3)-\varepsilon} E_{\nu}^{\text {pair }}=-\left(\frac{T}{12}+\frac{S}{12}\right) N_{\nu=8 / 3}^{L=1} .
\end{gathered}
$$

Therefore, a valley in the energy spectrum appears as

$$
\Delta E_{\nu=8 / 3}^{\text {pair }}=E_{\nu=8 / 3}^{\text {pair }}-\lim _{\nu \rightarrow(8 / 3) \pm \varepsilon} E_{\nu}^{\text {pair }}=-\frac{1}{12}(T+S) N_{\nu=8 / 3}^{L=1} .
$$

The $v=14 / 5,18 / 7$, and $19 / 7$ states have the most uniform configuration as shown in Figures 16, 17, and 18, respectively. The allowed transitions are schematically drawn by the black and green arrow pairs for the first and second nearest electron pairs, respectively.

The number of the allowed transitions is $1 / 5$ times the number of Landau orbitals with $L=1$. Then, the pair energy of $\mathrm{AB}$ and $\mathrm{CD}$ is given, respectively, by

$$
\begin{array}{ll}
\varsigma_{\mathrm{AB}}=-\left(\frac{1}{5}\right) T & \text { for } v=\frac{14}{5}, \\
\varsigma_{\mathrm{CD}}=-\left(\frac{1}{5}\right) S & \text { for } \nu=\frac{14}{5} .
\end{array}
$$

There are many electron pairs represented by $\mathrm{AB}$ and $\mathrm{CD}$. The total number of the pairs represented by $A B$ is equal to 


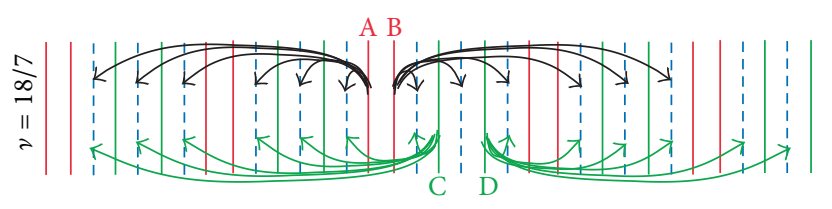

FIGURE 14: Quantum transitions at $v=18 / 7$ state.

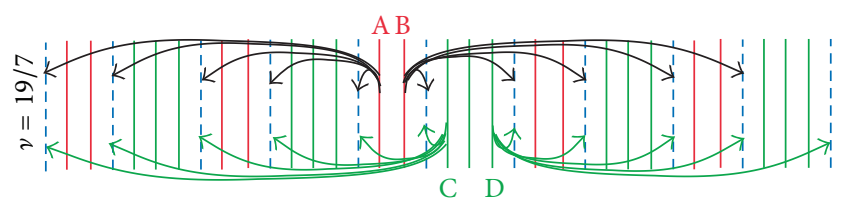

FIgURE 15: Quantum transitions at $v=19 / 7$ state.
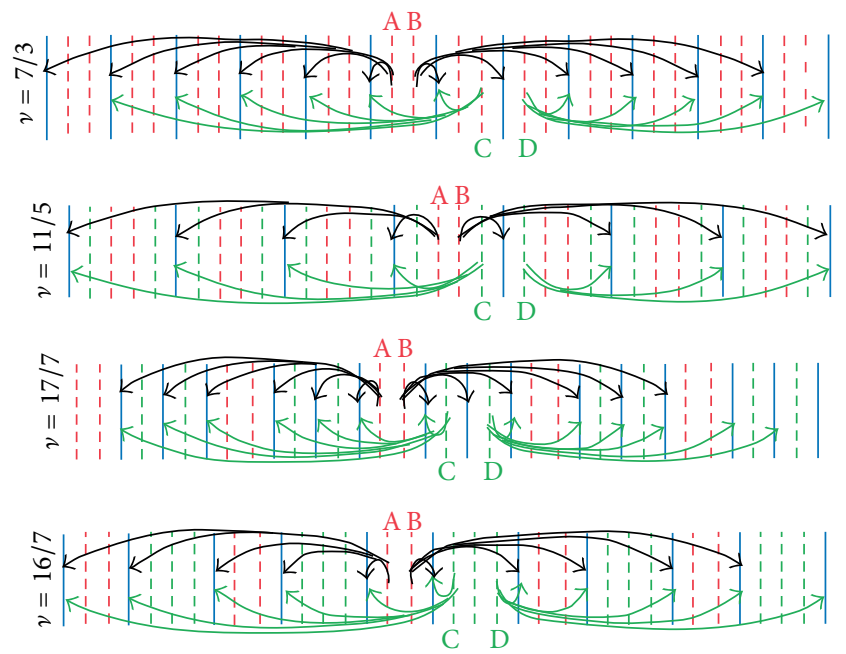

FIGURE 16: Quantum transitions at $v=7 / 3,11 / 5,17 / 7$, and $16 / 7$ states. Dashed lines indicate empty Landau orbitals with $L=1$ and solid lines are orbitals filled with electron.

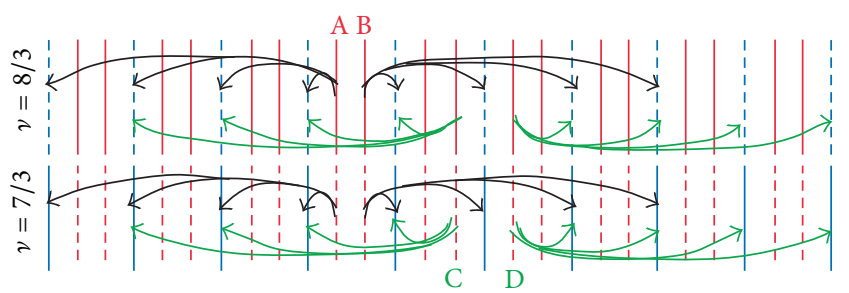

FIGURE 17: Comparison of allowed transitions between $v=8 / 3$ and $7 / 3$.

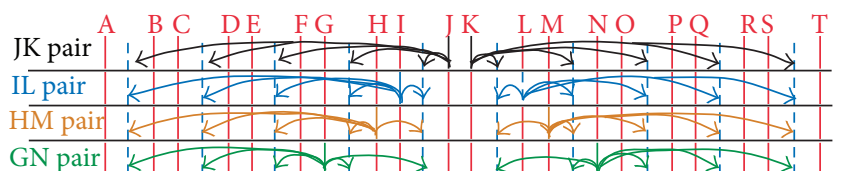

FIGURE 18: Various electron pairs with the same total momentum at $v=8 / 3$. Dashed lines indicate empty orbitals and solid lines indicate filled orbitals in the Landau level $L=1$. Allowed transitions from the electrons $J$ and $\mathrm{K}$ are shown by black arrow pairs, from IL by blue, from HM by brown, and from GN by dark green.
$(1 / 4) N_{\nu=14 / 5}^{L=1}$ and also that by CD is equal to $(1 / 4) N_{\nu=14 / 5}^{L=1}$. Then, we obtain the total pair energy for the electron pairs placed in the first and second neighboring Landau orbitals with $L=1$ as

$$
E_{\gamma=14 / 5}^{\text {pair }}=-\left(\frac{T}{20}+\frac{S}{20}\right) N_{\gamma=14 / 5}^{L=1} .
$$

Figure 14 shows the allowed transitions of the pairs $A B$ and CD at the filling factor $v=18 / 7$. The number of the empty orbitals is $3 / 7$ times that of the Landau orbitals with $L=1$. Accordingly, the pair energy of $\mathrm{AB}$ and $\mathrm{CD}$ is given, respectively, by

$$
\begin{array}{ll}
\varsigma_{\mathrm{AB}}=-\left(\frac{3}{7}\right) T & \text { for } v=\frac{18}{7} \\
\varsigma_{\mathrm{CD}}=-\left(\frac{3}{7}\right) S & \text { for } \nu=\frac{18}{7} .
\end{array}
$$

Then we obtain

$$
E_{\nu=18 / 7}^{\text {pair }}=-\left(\frac{3 T}{28}+\frac{3 S}{28}\right) N_{\nu=18 / 7}^{L=1}
$$

Next we count the number of allowed transitions of the pairs $\mathrm{AB}$ and $\mathrm{CD}$ at $v=19 / 7$. The electron pairs $\mathrm{AB}$ and $\mathrm{CD}$ in Figure 15 can transfer to all the empty Landau orbitals with $L=1$.

Since the number of the allowed transitions for each of the $\mathrm{AB}$ and $\mathrm{CD}$ pairs is two per unit configuration composed of the seven Landau orbitals, the pair energy of $A B$ and $C D$ is given, respectively, by

$$
\begin{array}{ll}
\varsigma_{\mathrm{AB}}=-\left(\frac{2}{7}\right) T & \text { for } v=\frac{19}{7} \\
\varsigma_{\mathrm{CD}}=-\left(\frac{2}{7}\right) S & \text { for } \nu=\frac{19}{7} .
\end{array}
$$

Then we obtain

$$
E_{v=19 / 7}^{\text {pair }}=-\left(\frac{2 T}{35}+\frac{2 S}{35}\right) N_{v=19 / 7}^{L=1}
$$

Thus, the electron pairs $\mathrm{AB}$ and $\mathrm{CD}$ can transfer to all the empty orbitals at $v=5 / 2,8 / 3,14 / 5,18 / 7$, and 19/7, and therefore the pair energy becomes very low, resulting in a strong binding energy.

The values of $S$ and $T$ may vary from sample to sample. We examine the condition that the $5 / 2$ plateau appears. From (38), the $v=5 / 2$ state is stable when $S$ is sufficiently lager than $T / 9$. In the experiment [9], the energy gaps have been measured as in Figure 4. In the high mobility sample [9], the energy gap at $v=5 / 2$ is nearly equal to that at $v=8 / 3$. Equations (21) and (41b) give the pair energy per electron as follows:

$$
\begin{gathered}
\frac{E_{\nu=5 / 2}^{\text {pair }}}{N_{\nu=5 / 2}^{L=1}}=-\left(\frac{S}{2}\right), \\
\frac{E_{\nu=8 / 3}^{\text {pair }}}{N_{\nu=8 / 3}^{L=1}}=-\left(\frac{T}{6}+\frac{S}{6}\right) .
\end{gathered}
$$


The experimental data [9] can be explained by the present theory under the following condition:

$$
T \approx 2 S \text {. }
$$

Equations (21), (41b), (45), and (49) give the theoretical ratio of the pair energies at $\nu=5 / 2,8 / 3,14 / 5$, and $19 / 7$ as follows:

$$
\begin{aligned}
& \left|\frac{E_{\nu=5 / 2}^{\text {pair }}}{N_{\nu=5 / 2}^{L=1}}\right|:\left|\frac{E_{\nu=8 / 3}^{\text {pair }}}{N_{\nu=8 / 3}^{L=1}}\right|:\left|\frac{E_{\nu=14 / 5}^{\text {pair }}}{N_{\nu=14 / 5}^{L=1}}\right|:\left|\frac{E_{\nu=19 / 7}^{\text {pair }}}{N_{\nu=19 / 7}^{L=1}}\right| \\
& =\left(\frac{S}{2}\right):\left(\frac{T}{6}+\frac{S}{6}\right):\left(\frac{T}{20}+\frac{S}{20}\right):\left(\frac{2 T}{35}+\frac{2 S}{35}\right) .
\end{aligned}
$$

When condition (51) is satisfied, the theoretical ratio of the pair energies becomes

$$
\begin{aligned}
& \left(\frac{S}{2}\right):\left(\frac{T}{6}+\frac{S}{6}\right):\left(\frac{T}{20}+\frac{S}{20}\right):\left(\frac{2 T}{35}+\frac{2 S}{35}\right) \\
& =1: 1:\left(\frac{3}{10}\right):\left(\frac{12}{35}\right) .
\end{aligned}
$$

From Figure 4, the experimental data of the energy gap yield the ratio for the high mobility sample as

$$
\begin{aligned}
& 0.0047: 0.005: 0.0023: 0.001 \\
& \quad=0.94: 1: 0.46: 0.2 .
\end{aligned}
$$

Thus, the present theory explains reasonably well the experimental data.

\section{FQH States at Filling Factors $2<v<2.5$}

Next we examine the FQH states with $2<v<2.5$. The most uniform configurations at $\nu=7 / 3,11 / 5,17 / 7$, and $16 / 7$ are schematically drawn in Figure 16. The hole-pairs AB and CD can transfer to all the electron states in $L=1$ as easily seen in Figure 16. This property produces a strong binding energy between the hole-pairs.

The number of allowed transitions for the hole-pairs at $v=7 / 3$ is equal to that for the electron-pairs at $v=8 / 3$. This symmetry between electron and hole is clearly seen by comparing the number of transitions (namely, number of arrows) for $\nu=8 / 3$ and 7/3, as easily seen in Figure 17.

From the discussion given in Sections 3, 5, and 6, we find that the state with $v=5 / 2,7 / 3,8 / 3,11 / 5,14 / 5,16 / 7,17 / 7$, and $18 / 7$ and $19 / 7$ is stable in the region $2<v<3$. The left panel of Figure 2 quoted from [5] shows the plateaus of the Hall resistance at $v=5 / 2,7 / 3,8 / 3,11 / 5,14 / 5$, and 16/7. Similar investigation for $\nu>3$ can be performed by using the method of this section. The pair energy becomes large at the filling factor $v=7 / 2,10 / 3,11 / 3,16 / 5$, and so on. The Hall plateaus at these filling factors have been found in several experiments.

The number of the allowed transitions via the Coulomb interaction discontinuously varies with changing the filling factor. The discontinuous variation is caused by the combined effect of the momentum conservation along the current, the most uniform configuration of electrons and the Fermi-Dirac statistics. This effect produces the stability of FQH states at the several filling factors.

As described in Section 1, different states have been proposed by different authors. For example, the $v=5 / 2 \mathrm{FQH}$ state is explained by the stripe HR or MR states and so on. The $v=7 / 3 \mathrm{FQH}$ state is said to be composed of the composite fermions where each electron binds to two flax quanta and the $v=11 / 5 \mathrm{FQH}$ state is explained to be composed of the composite fermions where each electron binds to four flax quanta. The theory presented here explains the FQH phenomena occurring at $v<2$ and those at $2<v<3$ in a coherent way without assuming any quasi-particles.

\section{Further Investigation of the Pair Energy for $2<v<3$}

We examine the exact energy of any FQH state. The total energy $E_{T}$ of the quasi-2D electron system is the sum of the eigenenergy $W$ of $H_{D}$ and the pair energy $E^{\text {pair }}$ via the interaction $H_{I}$ as follows:

$$
E_{T}=W+E^{\text {pair }}
$$

where $W$ has been already given by (10) as follows:

$$
W=\sum_{i=1}^{N} E_{L_{i}}\left(p_{i}\right)+C\left(L_{1}, \ldots, L_{N} ; p_{1}, \ldots, p_{N}\right) .
$$

Equations (5) and (6) yield the following equation:

$$
\begin{aligned}
W= & C\left(L_{1}, \ldots, L_{N} ; p_{1}, \ldots, p_{N}\right)+N \lambda \\
& +\sum_{i=1}^{N} U\left(\frac{p_{i}}{(e B)}\right)+\sum_{i=1}^{N}\left(\frac{\hbar e B}{m^{*}}\right)\left(L_{i}+\frac{1}{2}\right) .
\end{aligned}
$$

The energy difference between different Landau levels is extremely large as shown in (22) and (23). The interval of Landau energies has been estimated for GaAs

$$
\frac{\left(E_{1}-E_{0}\right)}{k_{B}}=\frac{\left(E_{2}-E_{1}\right)}{k_{B}} \approx 80.3[\mathrm{~K}] \text { for } B=4 T \text {. }
$$

The experimental values of the energy gaps at $2<v<3$ are smaller than $1[\mathrm{~K}]$ as in Table 1 and therefore higher Landau levels can be ignored at low temperatures. So, the ground state at $2<v<3$ is obtained by superposing many-electron states that all the Landau states with $L=0$ are occupied by electrons with up and down spins and the Landau states with $L=1$ are partially occupied by electrons. We express the number of electrons in the Landau level L by $N_{v}^{L}$ and the number of 
Landau orbitals by $N^{\text {level } L}$, respectively. The ratio $N_{v}^{L} / N^{\text {level } L}$ is described as follows:

$$
\frac{N_{v}^{L=0}}{N^{\text {levelL }}}=2 \text { in the ground state with } 2<v<3,
$$

$\frac{N_{v}^{L=1}}{N^{\text {levelL }}}=v-2 \quad$ in the ground state with $2<v<3$,

$$
\frac{N_{v}^{L=2}}{N^{\text {level } L}}=0 \quad \text { in the ground state with } 2<v<3 \text {, }
$$

$$
\frac{N_{\nu}^{L>2}}{N^{\text {levelL }}}=0 \quad \text { in the ground state with } 2<v<3,
$$

where $N^{\text {levelL }}$ depends on the sample and the magnetic field strength but is independent of $L$. The total number $N$ of electrons is

$$
N=N_{v}^{L=0}+N_{v}^{L=1} \text { in the ground state with } 2<v<3 .
$$

Substitution of (59a), (59b), (59c), (59d), and (60) into (57) gives the eigenenergy $W$ of $H_{D}$ as follows:

$$
\begin{aligned}
W= & C\left(L_{1}, \ldots, L_{N} ; p_{1}, \ldots, p_{N}\right)+N \lambda \\
& +\sum_{i=1}^{N} U\left(\frac{p_{i}}{(e B)}\right)+\frac{1}{2}\left(\frac{\hbar e B}{m^{*}}\right) N_{v}^{L=0} \\
& +\frac{3}{2}\left(\frac{\hbar e B}{m^{*}}\right) N_{v}^{L=1} \quad \text { in the ground state with } 2<v<3 .
\end{aligned}
$$

Next, we investigate the pair energy which is caused by the quantum transitions via $H_{I}$. The electron pairs in the ground state with $2<v<3$ have been classified into the following three types:

First type: both electrons in the pair are placed in the orbitals with $L=0$ only.

Second type: one electron is placed in $L=0$ and the other in $L=1$.

Third type: both electrons in the pair are placed in $L=$ 1 only.

These pair energies are described by the symbols $E_{L=0}^{\text {pair }}$, $E_{L=0 \text { and } 1}^{\text {pair }}$, and $E_{L=1}^{\text {pair }}$, respectively. The total energy of all the electron pairs is

$$
\begin{aligned}
E^{\text {pair }}= & E_{L=0}^{\text {pair }}+E_{L=0 \text { and } 1}^{\text {pair }} \\
& +E_{L=1}^{\text {pair }} \quad \text { in the ground state with } 2<v<3 .
\end{aligned}
$$

Therein the pair energies $E_{L=0}^{\text {pair }}$ and $E_{L=0 \text { and } 1}^{\text {pair }}$ are negligibly small because of the following reason. Any order of the perturbation energy is obtained by a summation of the functions with the denominator containing the energy difference of $W$ between the ground and intermediate states. Any electron pair belonging to the first or second types can transfer only to the intermediate states with a higher Landau level because all the Landau orbitals with $L=0$ are already occupied by electrons with up and down spins. Therefore, the energy difference between the ground and intermediate states is very large as in (58). Then we may ignore the pair energy belonging to the first and second types:

$E_{L=0}^{\text {pair }} \approx 0, \quad E_{L=0 \text { and } 1}^{\text {pair }} \approx 0 \quad$ in the ground state with $\nu>2$.

On the other hand, the electron pairs in $L=1$ can transfer to empty orbitals with $L=1$ for $2<v<3$. Then, the energy difference of $W$ between the ground and the intermediate states comes from the difference in the classical Coulomb energies and so the difference is very small. We will examine any electron (or hole) pair placed in any Landau orbitals with $L=1$. As an example, we discuss the case of $v=8 / 3$. Figure 18 schematically shows the electron pairs at $v=8 / 3$. The electron pairs IL, HM, and GN possess the total momentum same as that of the pair JK. These pairs can transfer to all the empty orbitals as easily seen in Figure 18.

The momenta of the electrons at G, H, I, J, K, L, M, and $\mathrm{N}$ are described by the symbols $p_{\mathrm{G}}, p_{\mathrm{H}}, p_{\mathrm{I}}, p_{\mathrm{J}}, p_{\mathrm{K}}, p_{\mathrm{L}}, p_{\mathrm{M}}$, and $p_{\mathrm{N}}$, respectively. Then, the total momenta of the electron pairs take the same value because of (4) and (5):

$$
p_{\text {total }}=p_{\mathrm{G}}+p_{\mathrm{N}}=p_{\mathrm{H}}+p_{\mathrm{M}}=p_{\mathrm{I}}+p_{\mathrm{L}}=p_{\mathrm{J}}+p_{\mathrm{K}} .
$$

The energies of the pairs GN, HM, IL, and JK, $\varsigma_{\mathrm{GN}}, \varsigma_{\mathrm{HM}}, \varsigma_{\mathrm{IL}}$, and $\varsigma_{\mathrm{JK}}$, can be reexpressed systematically by using a symbol $\varsigma_{v}^{L=1}\left(p_{\text {total }}, j\right)$, where $p_{\text {total }}$ and $j$ indicate the total momentum and the distance between the pair as follows:

$$
\begin{gathered}
\varsigma_{\mathrm{JK}}=\varsigma_{\nu=8 / 3}^{L=1}\left(p_{\text {total }}, j=1\right), \\
\varsigma_{\mathrm{IL}}=\varsigma_{\nu=8 / 3}^{L=1}\left(p_{\text {total }}, j=5\right), \\
\varsigma_{\mathrm{HM}}=\varsigma_{\nu=8 / 3}^{L=1}\left(p_{\text {total }}, j=7\right), \\
\varsigma_{\mathrm{GN}}=\varsigma_{\nu=8 / 3}^{L=1}\left(p_{\text {total }}, j=11\right) .
\end{gathered}
$$

Therein the momentum of each electron is given as

$$
\begin{aligned}
& p_{\mathrm{J}}=\frac{1}{2}\left(p_{\text {total }}-\frac{1 \times 2 \pi \hbar}{\ell}\right), \\
& p_{\mathrm{K}}=\frac{1}{2}\left(p_{\text {total }}+\frac{1 \times 2 \pi \hbar}{\ell}\right), \\
& p_{\mathrm{I}}=\frac{1}{2}\left(p_{\text {total }}-\frac{5 \times 2 \pi \hbar}{\ell}\right), \\
& p_{\mathrm{L}}=\frac{1}{2}\left(p_{\text {total }}+\frac{5 \times 2 \pi \hbar}{\ell}\right),
\end{aligned}
$$




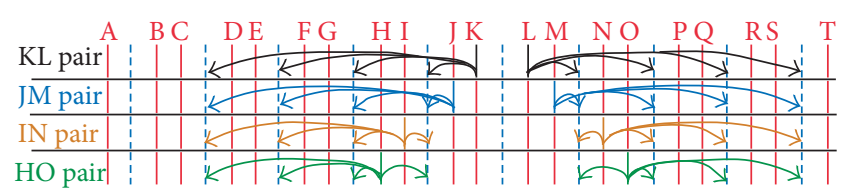

FIGURE 19: Various electron pairs with the same total momentum at $\nu=8 / 3$. Dashed lines indicate empty orbitals and solid lines indicate filled orbitals in the Landau level $L=1$. Allowed transitions from the electrons $\mathrm{K}$ and $\mathrm{L}$ are shown by black arrow pairs, from JM by blue, from IN by brown, and from $\mathrm{HO}$ by dark green.

$$
\begin{aligned}
& p_{\mathrm{H}}=\frac{1}{2}\left(p_{\text {total }}-\frac{7 \times 2 \pi \hbar}{\ell}\right), \\
& p_{\mathrm{M}}=\frac{1}{2}\left(p_{\text {total }}+\frac{7 \times 2 \pi \hbar}{\ell}\right), \\
& p_{\mathrm{G}}=\frac{1}{2}\left(p_{\text {total }}-\frac{11 \times 2 \pi \hbar}{\ell}\right), \\
& p_{\mathrm{N}}=\frac{1}{2}\left(p_{\text {total }}+\frac{11 \times 2 \pi \hbar}{\ell}\right) .
\end{aligned}
$$

Thus, any momentum-pair $\left(p_{V}, p_{W}\right)$ is related to $p_{\text {total }}$ and $j$ as

$$
\begin{gathered}
p_{V}=\frac{1}{2}\left(p_{\text {total }}-\frac{j \times 2 \pi \hbar}{\ell}\right), \\
p_{W}=\frac{1}{2}\left(p_{\text {total }}+\frac{j \times 2 \pi \hbar}{\ell}\right) . \\
p_{\text {total }}=p_{V}+p_{W}, \quad j=\frac{\left(p_{W}-p_{V}\right)}{(2 \pi \hbar / \ell)} .
\end{gathered}
$$

Because both momenta $p_{V}$ and $p_{W}$ should be equal to $(2 \pi \hbar / l) \times$ integer, the values of $p_{\text {total }}$ and $j$ are classified to the following two cases:

$$
\begin{aligned}
& p_{\text {total }}=\left(\frac{2 \pi \hbar}{\ell}\right) \times(\text { odd integer }) \quad \text { for } j=(\text { odd integer }), \\
& p_{\text {total }}=\left(\frac{2 \pi \hbar}{\ell}\right) \times(\text { even integer }) \text { for } j=(\text { even integer }) .
\end{aligned}
$$

We have already examined the case of odd integer $j$ in Figure 18.

Next, we examine the case of even integer $j$. Figure 19 shows quantum transitions with even integers $j$ given by (68b). All the electron pairs possessing the total momentum same as that of the pair KL can transfer to all the empty orbitals as in Figure 19. The electron pairs KL, JM, IN, and $\mathrm{HO}$ indicate the cases of $j=2,4,8$, and 10 , respectively.
The pair energies are described as

$$
\begin{gathered}
\varsigma_{\mathrm{KL}}=\varsigma_{v=8 / 3}^{L=1}\left(p_{\text {total }}, j=2\right), \\
\varsigma_{\mathrm{JM}}=\varsigma_{v=8 / 3}^{L=1}\left(p_{\text {total }}, j=4\right), \\
\varsigma_{\mathrm{IN}}=\varsigma_{\nu=8 / 3}^{L=1}\left(p_{\text {total }}, j=8\right), \\
\varsigma_{\mathrm{HO}}=\varsigma_{\nu=8 / 3}^{L=1}\left(p_{\text {total }}, j=10\right) .
\end{gathered}
$$

The total energy of all the electron pairs is described by the symbol $E^{\text {pair }}$ defined by (55). Use of (62) and (63) gives

$$
E^{\text {pair }} \approx E_{L=1}^{\text {pair }}(\nu) \quad \text { in the ground state with } 2<v<3 .
$$

This energy at $L=1$ is the sum of all the pair energies with $p_{\text {total }}$ and $j$ :

$E_{L=1}^{\text {pair }}(\nu)$

$$
=\sum_{p_{\text {total }}, j} \varsigma_{v}^{L=1}\left(p_{\text {total }}, j\right) \quad \text { in the ground state with } 2<v<3 .
$$

Equations (55), (70), and (71) yield the total energy of the quasi-2D electron system as follows:

$$
E_{T} \approx W+\sum_{p_{\text {total }}, j} \varsigma_{\nu}^{L=1}\left(p_{\text {total }}, j\right)
$$

in the ground state with $2<v<3$.

Substitution of (61) into (72) gives

$$
\begin{aligned}
E_{T} \approx & C\left(L_{1}, \ldots, L_{N} ; p_{1}, \ldots, p_{N}\right) \\
& +N \lambda+\sum_{i=1}^{N} U\left(\frac{p_{i}}{(e B)}\right)+\frac{1}{2}\left(\frac{\hbar e B}{m^{*}}\right) N_{v}^{L=0} \\
& +\frac{3}{2}\left(\frac{\hbar e B}{m^{*}}\right) N_{v}^{L=1}+\sum_{p_{\text {total }}, j} \varsigma_{v}^{L=1}\left(p_{\text {total }}, j\right)
\end{aligned}
$$

in the ground state with $2<v<3$.

We express the pair energy per electron by the symbol $\xi_{v}^{L=1}(j)$ which is defined by

$$
\begin{aligned}
& \xi_{v}^{L=1}(j) \\
& \quad=\frac{\sum_{p_{\text {total }}} \varsigma_{v}^{L=1}\left(p_{\text {total }}, j\right)}{N_{v}^{L=1}} \text { in the ground state with } 2<v<3 .
\end{aligned}
$$

The exact pair energy is the sum of all order terms in the perturbation calculation as follows:

$$
\xi_{\nu}^{L=1}(j)=\sum_{n=2,3,4, \ldots} \xi_{\nu}^{L=1}(j ; n)
$$


TABLE 2: Second order of the perturbation energy per electron for the electron pairs placed in the second nearest Landau orbital pairs.

\begin{tabular}{|c|c|c|c|c|c|c|c|}
\hline$v$ & $5 / 2$ & $48 / 19$ & $78 / 31$ & $8 / 3$ & $14 / 5$ & $18 / 7$ & $19 / 7$ \\
\hline$\xi_{v}^{L=1}(2 ; 2)$ & $-(1 / 2) S$ & $-(41 / 190) S$ & $-(113 / 496) S$ & $-S / 6$ & $-S / 20$ & $-3 S / 28$ & $-2 S / 35$ \\
\hline
\end{tabular}

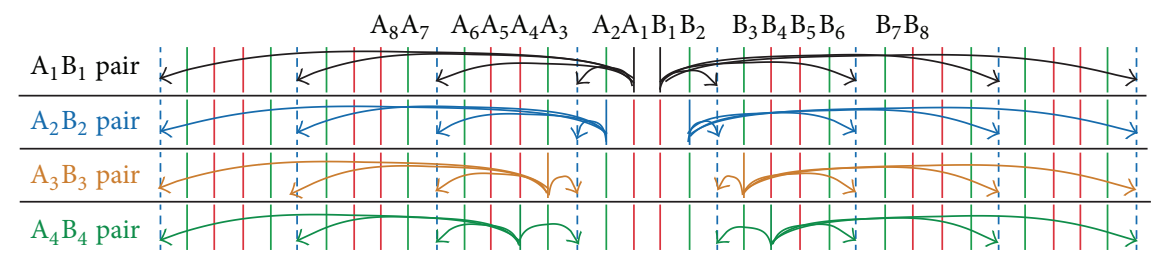

FIGURE 20: Various electron pairs with the same total momentum at $v=14 / 5$. Dashed lines indicate empty orbitals and solid lines indicate filled orbitals in the Landau level $L=1$. Allowed transitions from the electrons $\mathrm{A}_{1}$ and $\mathrm{B}_{1}$ are shown by black arrow pairs, from $\mathrm{A}_{2} \mathrm{~B}_{2}$ by blue, from $\mathrm{A}_{3} \mathrm{~B}_{3}$ by brown, and from $\mathrm{A}_{4} \mathrm{~B}_{4}$ by dark green.

where $\xi_{v}^{L=1}(j ; n)$ indicates the $n$th order of the perturbation energy. Substitution of (74) and (75) into (73) yields

$$
\begin{aligned}
E_{T}= & W+E^{\text {pair }} \\
\approx & C\left(L_{1}, \ldots, L_{N} ; p_{1}, \ldots, p_{N}\right)+N \lambda \\
& +\sum_{i=1}^{N} U\left(\frac{p_{i}}{(e B)}\right)+\frac{1}{2}\left(\frac{\hbar e B}{m^{*}}\right) N_{v}^{L=0} \\
& +\frac{3}{2}\left(\frac{\hbar e B}{m^{*}}\right) N_{v}^{L=1}+N_{v}^{L=1} \\
& \times \sum_{j=1,2,3, \ldots}\left(\sum_{n=2,3,4, \ldots} \xi_{v}^{L=1}(j ; n)\right)
\end{aligned}
$$

in the ground state with $2<v<3$.

Therein the function form of $W$ is continuous with the change in $\nu$. On the other hand, the pair energy $E^{\text {pair }}$ has a discontinuous form for the argument $\nu$, because the number of the allowed transitions depends discontinuously upon $\nu$. This discontinuous property produces the plateaus of the Hall resistance at specific filling factors. We have already calculated the second order perturbation energies for $j=1$ and 2 as in (21), (31), (37), (41b), (45), (47), and (49). We list the results in Tables 2 and 3.

Now we examine the effects of further neighbouring electron pairs in the $v=14 / 5$ state. Figure 20 shows the most uniform configuration. Therein the allowed transitions from the electron pairs $\mathrm{A}_{n} \mathrm{~B}_{n}(n=1,2,3, \ldots)$ are shown by the arrow pairs. The centre position between the nearest pair $\mathrm{A}_{1} \mathrm{~B}_{1}$ is equal to that of the electron pair $\mathrm{A}_{n} \mathrm{~B}_{n}$ for any integer $n>1$. Accordingly, the total momentum of the pair $A_{n} B_{n}$ is equal to that of the pair $A_{1} B_{1}$. Therefore, the electron pair $\mathrm{A}_{n} \mathrm{~B}_{n}$ with $L=1$ can transfer to all the empty states as the pair $\mathrm{A}_{1} \mathrm{~B}_{1}$.

Also, the total momentum of the electron pair $\mathrm{C}_{1} \mathrm{D}_{1}$ in Figure 21 is equal to that of the pairs $\mathrm{C}_{n} \mathrm{D}_{n}(n=1,2,3, \ldots)$ and therefore the pair $\mathrm{C}_{n} \mathrm{D}_{n}$ can transfer to all the empty states except the orbital $Y$ shown in blue. This only one forbidden transition may be ignored in comparison with the enormously many allowed transitions which are caused by the spreading of the Landau wave function in the $y$-direction for the macroscopic size of the device.

Thus, the further neighbouring electron (or hole) pairs with $j \geq 3$ can transfer to all the empty (or filled) orbitals at $\nu=8 / 3,14 / 5,7 / 3$, and $11 / 5$. The energies of these pairs with $j \geq 3$ are negative in the second order perturbation. Therefore, the energies are accumulated to give a stronger binding energy and so the states become more stable.

\section{Conclusions}

The FQH states with $2<v<3$ have been investigated by using the method developed in previous articles [25-32]. We have found the most uniform configuration in the Landau orbitals at $v=5 / 2,48 / 19,78 / 31,8 / 3,14 / 5,18 / 7,19 / 7,7 / 3$, $11 / 5,17 / 7$, and $16 / 7$. Especially, the electron (or hole) pairs placed in the first and second neighbouring Landau orbitals in $L=1$ can transfer to all the empty orbitals at $\nu_{0}=$ $8 / 3,14 / 5,7 / 3$, and $11 / 5$ states via the Coulomb interaction. Also, at $v_{0}=5 / 2$, the electron pairs placed in the second nearest Landau orbitals can transfer to all the empty orbitals with $L=1$. More distant electron (or hole) pairs with the same centre positions as in the first and second nearest pairs can also transfer to all the empty (or filled) orbitals at $\nu_{0}$. Then, the energies of the distant pairs, $\xi_{v}^{L=1}(j)$ for $j \geq 3$, are accumulated to that of the first and second nearest pairs.

This capability of the quantum transitions to all the empty orbitals means that the number of transitions is largest at $v_{0}$. Accordingly, the number of transitions decreases abruptly when the filling factor deviates from $\nu_{0}=5 / 2,8 / 3,14 / 5,7 / 3$, and $11 / 5$. This property is caused by the combined effect of the most uniform configuration, momentum conservation and Fermi-Dirac statistics. For example, (41a), (41b)-(43) show that the pair energy at $v=8 / 3$ becomes half of that in the neighbourhood of $v=8 / 3$. The spectrum of the pair energy has a valley structure at $\nu_{0}=5 / 2,8 / 3,14 / 5,7 / 3,11 / 5$ and so on. That is to say, the pair energy has a discontinuous function form which takes the lowest value at the specific filling factor $v_{0}$ and becomes higher energy with a gap in the neighbourhood of $v_{0}$. Therefore, the $v_{0}$-FQH states are 


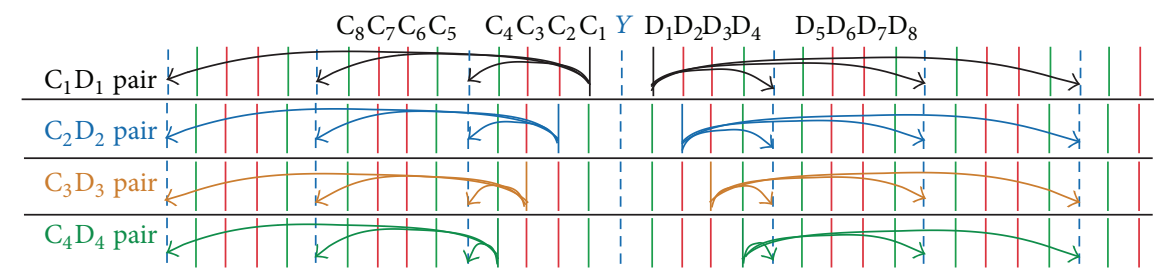

FIGURE 21: Various electron pairs with the same total momentum at $\nu=14 / 5$. Dashed lines indicate empty orbitals and solid lines indicate filled orbitals in the Landau level $L=1$. Allowed transitions from the electrons $C_{1}$ and $D_{1}$ are shown by black arrow pairs, from $C_{2} D_{2}$ by blue, from $\mathrm{C}_{3} \mathrm{D}_{3}$ by brown, and from $\mathrm{C}_{4} \mathrm{D}_{4}$ by dark green.

TABLE 3: Second order of the perturbation energy per electron for the electron pairs placed in the nearest Landau orbital pairs.

\begin{tabular}{lccccccc}
\hline$\nu$ & $5 / 2$ & $48 / 19$ & $78 / 31$ & $8 / 3$ & $14 / 5$ & $18 / 7$ & $19 / 7$ \\
\hline$\xi_{\nu}^{L=1}(1 ; 2)$ & 0 & $-(9 / 190) T$ & $-(15 / 496) T$ & $-T / 6$ & $-T / 20$ & $-3 T / 28$ & $-2 T / 35$ \\
\hline
\end{tabular}

stable at $\nu_{0}=5 / 2,8 / 3,14 / 5,7 / 3$, and $11 / 5$. Since thousands of the Landau wave functions are overlapping with each other, the deviation of the Hall resistance from $h /\left(\mathrm{e}^{2} v_{0}\right)$ becomes smaller than $0.1 \%$. This property is in agreement with the experimental value, the accuracy of which is $0.015 \%$ at $v_{0}=$ $7 / 2$ and so on. Thus, we should study the quasi-2D system with more than thousand electrons. Our treatment can do this task because the present theory can count the number of transitions for an enormous number of electrons.

When we choose the parameter-ratio $(T / S)=2$, the theoretical ratio of the pair energies at $\nu_{0}=5 / 2,8 / 3,14 / 5$, and $19 / 7$ is equal to $1: 1:(3 / 10):(12 / 35)$ which is in reasonable agreement with the data of the high mobility sample in [9].

The present theory has explained the FQH phenomena for various filling factors $2<v<3$ based on a standard treatment of interacting quasi-2D electron gas without assuming any quasiparticle.

\section{Conflict of Interests}

The author declares that there is no conflict of interests regarding the publication of this paper.

\section{Acknowledgments}

The author expresses his heartfelt appreciation for an important suggestion of Professor Klaus von Klitzing. He has suggested to the author to examine the $5 / 2$ plateau problem. Also the author wishes to express his appreciation for the encouragement of Professor Koichi Katsumata, Professor Masayuki Hagiwara, Professor Hidenobu Hori, and Professor Yasuyuki Kitano. Particularly Professor Katsumata has given his important suggestions for improving my description. The author could not have completed this paper without their support.

\section{References}

[1] W. Pan, R. R. Du, H. L. Stormer et al., "Strongly anisotropic electronic transport at Landau level filling factor $v=9 / 2$ and $\nu=5 / 2$ under a tilted magnetic field," Physical Review Letters, vol. 83, no. 4, pp. 820-823, 1999.

[2] M. P. Lilly, K. B. Cooper, J. P. Eisenstein, L. N. Pfeiffer, and K. W. West, "Evidence for an anisotropic state of two-dimensional electrons in high Landau levels," Physical Review Letters, vol. 82, no. 2, pp. 394-397, 1999.

[3] M. P. Lilly, K. B. Cooper, J. P. Eisenstein, L. N. Pfeiffer, and K. W. West, "Anisotropic states of two-dimensional electron systems in high Landau levels: effect of an in-plane magnetic field," Physical Review Letters, vol. 83, pp. 824-827, 1999.

[4] J. P. Eisenstein, K. B. Cooper, L. N. Pfeiffer, and K. W. West, "Insulating and fractional quantum hall states in the first excited Landau level," Physical Review Letters, vol. 88, Article ID 076801, 2002.

[5] C. R. Dean, B. A. Piot, P. Hayden et al., "Intrinsic gap of the $\nu=5 / 2$ fractional quantum hall state," Physical Review Letters, vol. 100, no. 14, Article ID 146803, 2008.

[6] J. S. Xia, W. Pan, C. L. Vicente et al., "Electron correlation in the second landau level: a competition between many nearly degenerate quantum phases," Physical Review Letters, vol. 93, no. 17, Article ID 176809, 2004.

[7] G. A. Csáthy, J. S. Xia, C. L. Vicente et al., "Tilt-induced localization and delocalization in the second Landau Level," Physical Review Letters, vol. 94, Article ID 146801, 2005.

[8] W. Pan, J. S. Xia, H. L. Stormer et al., "Experimental studies of the fractional quantum Hall effect in the first excited Landau level," Physical Review B, vol. 77, no. 7, Article ID 075307, 2008.

[9] H. C. Choi, W. Kang, S. Das Sarma, L. N. Pfeiffer, and K. W. West, "Activation gaps of fractional quantum Hall effect in the second Landau level," Physical Review B, vol. 77, no. 8, Article ID 081301, 2008.

[10] I. L. Aleiner and L. I. Glazman, "Two-dimensional electron liquid in a weak magnetic field," Physical Review B, vol. 52, no. 15, pp. 11296-11312, 1995.

[11] A. A. Koulakov, M. M. Fogler, and B. I. Shklovskii, "Charge density wave in two-dimensional electron liquid in weak magnetic field," Physical Review Letters, vol. 76, no. 3, pp. 499-502, 1996.

[12] R. Moessner and J. T. Chalker, "Exact results for interacting electrons in high Landau levels," Physical Review B, vol. 54, no. 7, pp. 5006-5015, 1996.

[13] E. H. Rezayi, F. D. M. Haldane, and K. Yang, "Charge-densitywave ordering in half-filled high Landau levels," Physical Review Letters, vol. 83, no. 6, pp. 1219-1222, 1999. 
[14] F. D. M. Haldane and E. H. Rezayi, "Spin-singlet wave function for the half-integral quantum Hall effect," Physical Review Letters, vol. 60, no. 10, pp. 956-959, 1988.

[15] G. Moore and N. Read, "Nonabelions in the fractional quantum hall effect," Nuclear Physics B, vol. 360, pp. 362-396, 1991.

[16] N. Read, "Paired fractional quantum Hall states and the $v=5 / 2$ puzzle," Physica B, vol. 298, pp. 121-128, 2001.

[17] J. Bardeen, L. N. Cooper, and J. R. Schrieffer, "Theory of superconductivity," Physical Review, vol. 108, no. 5, pp. 11751204, 1957.

[18] J. Bardeen, L. N. Cooper, and J. R. Schrieffer, "Microscopic theory of superconductivity," Physical Review, vol. 106, pp. 162$164,1957$.

[19] M. Greiter, X. G. Wen, and F. Wilczek, "Paired Hall state at half filling," Physical Review Letters, vol. 66, pp. 3205-3208, 1991.

[20] M. Greiter, X. G. Wen, and F. Wilczek, "Paired Hall states," Nuclear Physics B, vol. 374, pp. 567-614, 1992.

[21] R. H. Morf, “Transition from quantum Hall to compressible states in the second Landau level: new light on the $v=5 / 2$ enigma," Physical Review Letters, vol. 80, pp. 1505-1508, 1998.

[22] E. H. Rezayi and F. D. M. Haldane, "Incompressible paired hall state, stripe order, and the composite fermion liquid phase in half-filled landau levels," Physical Review Letters, vol. 84, no. 20, pp. 4685-4688, 2000.

[23] R. Tao and D. J. Thouless, "Fractional quantization of Hall conductance," Physical Review B, vol. 28, pp. 1142-1144, 1983.

[24] R. Tao, "Fractional quantization of Hall conductance. II," Physical Review B, vol. 29, pp. 636-644, 1984.

[25] S. Sasaki, "Energy gap in fractional quantum Hall effect," Physica B, no. 281-282, pp. 838-839, 2000.

[26] S. Sasaki, "Binding energy and polarization of fractional quantum Hall state," in Proceedings of the 25th International Conference on the Physics of Semiconductors, pp. 925-926, Springer, 2000.

[27] S. Sasaki, "Spin polarization in fractional quantum Hall effect," Surface Science, vol. 532-535, pp. 567-575, 2003.

[28] S. Sasaki, "Spin Peierls effect in spin polarization of fractional quantum Hall states," in Proceedings of the 22nd European Conference on Surface Science, vol. 566-568, pp. 1040-1046, September 2004.

[29] S. Sasaki, Surface Science: New Research, pp. 103-161, chapter 4, Nova Science Publishers, 2005.

[30] S. Sasaki, "Energy gaps in fractional quantum Hall states," Journal of Physics, vol. 100, Article ID 042021, 4 pages, 2008.

[31] S. Sasaki, "Tunneling effect in quantum Hall device," e-Journal of Surface Science and Nanotechnology, vol. 8, pp. 121-124, 2010.

[32] S. Sasaki, "Gap structure and gapless structure in fractional quantum Hall effect," Advances in Condensed Matter Physics, vol. 2012, Article ID 281371, 13 pages, 2012.

[33] M. Büttiker, "Absence of backscattering in the quantum Hall effect in multiprobe conductors," Physical Review B, vol. 38, pp. 9375-9389, 1988.

[34] H. L. Stormer, D. C. Tsui, and A. C. Gossard, "The fractional quantum hall effect," Reviews of Modern Physics, vol. 71, pp. S298-S305, 1999.

[35] C. R. Dean, A. F. Young, P. Cadden-Zimansky et al., "Multicomponent fractional quantum Hall effect in graphene," Nature Physics, vol. 7, no. 9, pp. 693-696, 2011.

[36] D. K. Ki, V. I. Fal'ko, and A. F. Morpurgo, "Even denominator fractional quantum Hall state in multi-terminal suspended bilayer graphene devices," http://arxiv.org/abs/1305.4761.
[37] K. I. Bolotin, F. Ghahari, M. D. Shulman, H. L. Stormer, and P. Kim, "Observation of the fractional quantum Hall effect in graphene," Nature, vol. 462, no. 7270, pp. 196-199, 2009.

[38] D. R. Luhman, W. Pan, D. C. Tsui, L. N. Pfeiffer, K. W. Baldwin, and K. W. West, "Observation of a fractional quantum Hall state at $\nu=1 / 4$ in a wide GaAs quantum well," Physical Review Letters, vol. 101, Article ID 266804, 4 pages, 2008.

[39] J. Shabani, T. Gokmen, and M. Shayegan, "Correlated states of electrons in wide quantum wells at low fillings: the role of charge distribution symmetry," Physical Review Letters, vol. 103, Article ID 046805, 4 pages, 2009.

[40] J. Shabani, Y. Liu, M. Shayegan, L. N. Pfeiffer, K. W. West, and K. W. Baldwin, "Phase diagrams for the stability of the $v=1 / 2$ fractional quantum Hall effect in wide GaAs quantum wells," Physical Review B, vol. 88, no. 24, Article ID 245413, 8 pages, 2013. 

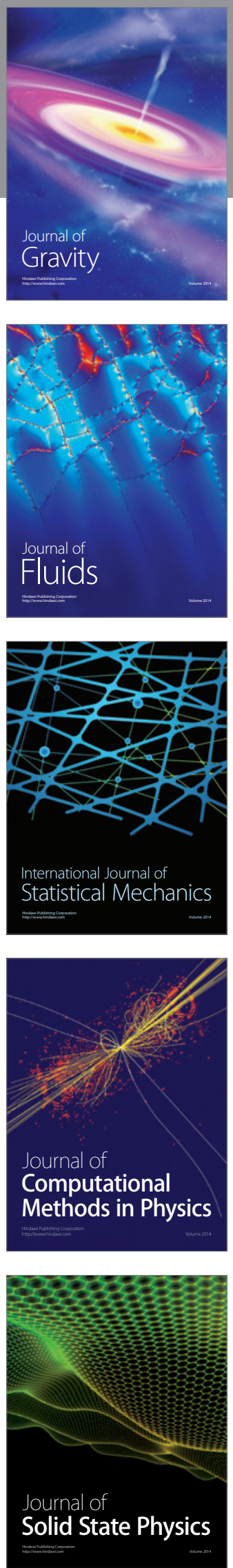

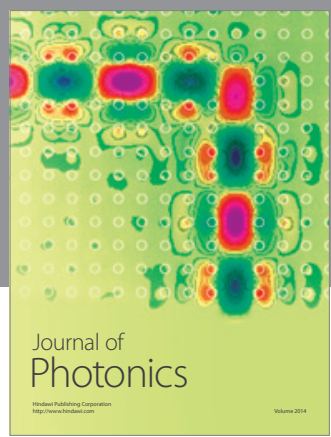

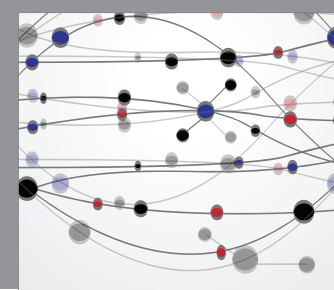

The Scientific World Journal

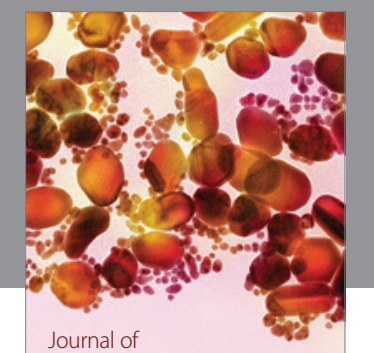

Soft Matter
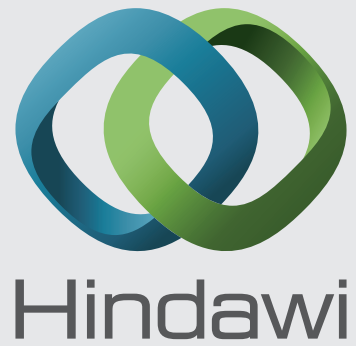

Submit your manuscripts at

http://www.hindawi.com
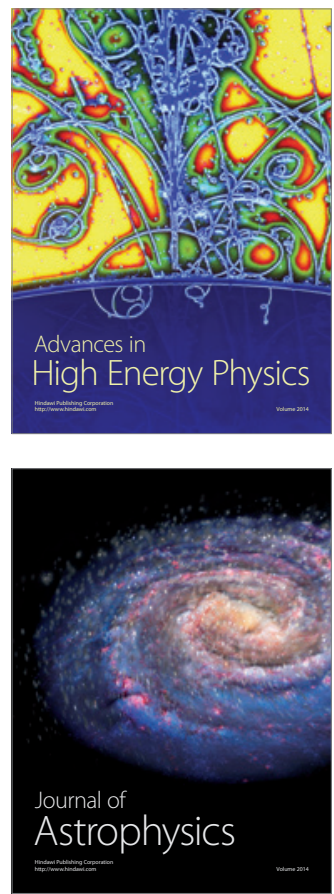
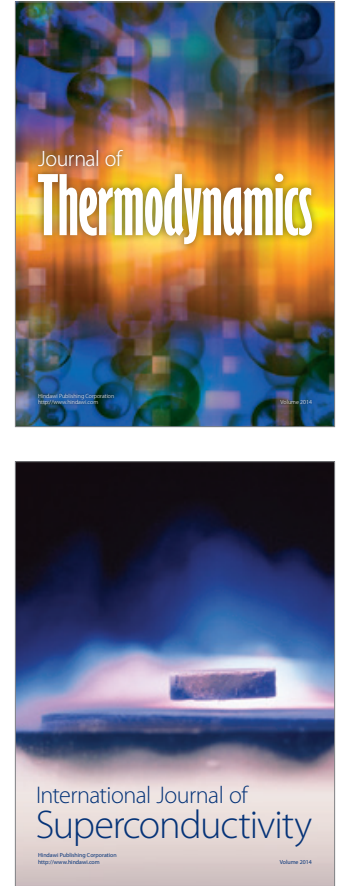
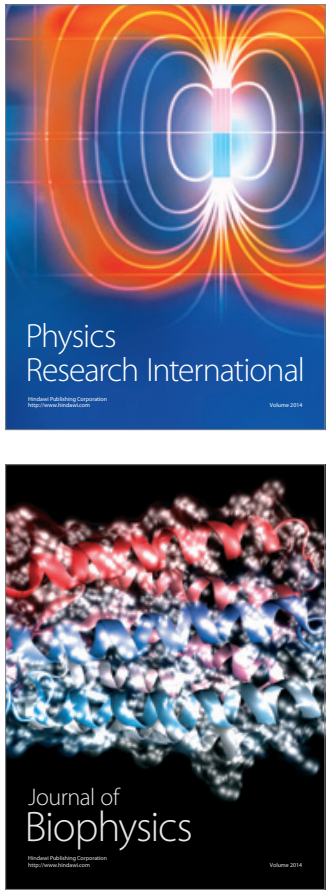
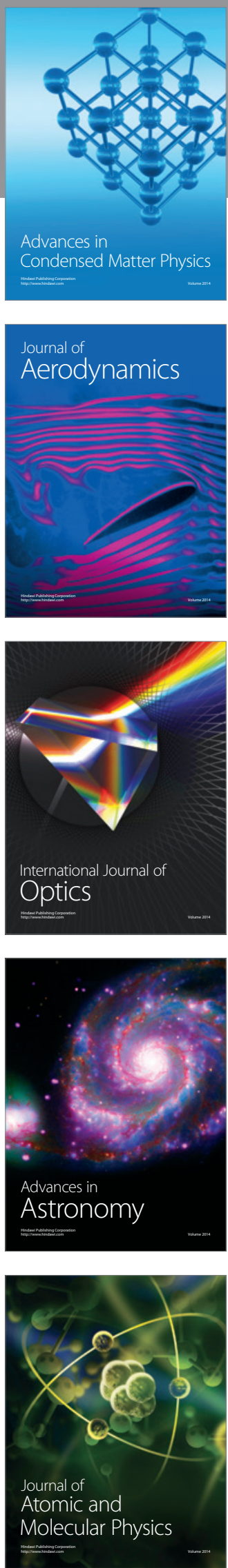